\title{
Identification of a nucleoside analog active against adenosine kinase-expressing plasma cell malignancies
}

\author{
Utthara Nayar, ${ }^{1}$ Jouliana Sadek, ${ }^{1}$ Jonathan Reichel, ${ }^{1}$ Denise Hernandez-Hopkins, ${ }^{1}$ Gunkut Akar, ${ }^{1}$ Peter J. Barelli, ${ }^{2}$ \\ Michelle A. Sahai, ${ }^{3}$ Hufeng Zhou, ${ }^{4}$ Jennifer Totonchy, ${ }^{1}$ David Jayabalan, ${ }^{1}$ Ruben Niesvizky, ${ }^{5}$ Ilaria Guasparri, ${ }^{1}$ Duane Hassane, ${ }^{5}$ \\ Yifang Liu, ${ }^{1}$ Shizuko Sei, ${ }^{6}$ Robert H. Shoemaker, ${ }^{7}$. David Warren, ${ }^{2}$ Olivier Elemento, ${ }^{3}$ Kenneth M. Kaye, ${ }^{4}$ and Ethel Cesarman ${ }^{1}$ \\ 1Department of Pathology and Laboratory Medicine, ${ }^{2}$ Department of Biochemistry, and ${ }^{3}$ Department of Physiology and Biophysics, Weill Cornell Medical College, New York, New York, USA \\ ${ }^{4}$ Department of Medicine, Brigham and Women's Hospital, and Harvard Medical School, Boston, Massachusetts, USA. 'Division of Hematology and Medical Oncology, Department of Medicine, \\ Weill Cornell Medical College, New York, New York, USA. ${ }^{6}$ Viral Vector Toxicology Section, Laboratory of Human Toxicology and Pharmacology, SAIC-Frederick, National Cancer Institute at Frederick, Frederick, \\ Maryland, USA. ${ }^{~ S}$ creening Technologies Branch, Developmental Therapeutics Program, Division of Cancer Treatment and Diagnosis, National Cancer Institute at Frederick, Frederick, Maryland, USA.
}

\begin{abstract}
Primary effusion lymphoma (PEL) is a largely incurable malignancy of B cell origin with plasmacytic differentiation. Here, we report the identification of a highly effective inhibitor of PEL. This compound, 6-ethylthioinosine (6-ETI), is a nucleoside analog with toxicity to PEL in vitro and in vivo, but not to other lymphoma cell lines tested. We developed and performed resistome analysis, an unbiased approach based on RNA sequencing of resistant subclones, to discover the molecular mechanisms of sensitivity. We found different adenosine kinase-inactivating (ADK-inactivating) alterations in all resistant clones and determined that ADK is required to phosphorylate and activate 6-ETI. Further, we observed that 6-ETI induces ATP depletion and cell death accompanied by $S$ phase arrest and DNA damage only in ADK-expressing cells. Immunohistochemistry for ADK served as a biomarker approach to identify 6-ETI-sensitive tumors, which we documented for other lymphoid malignancies with plasmacytic features. Notably, multiple myeloma (MM) expresses high levels of ADK, and 6-ETI was toxic to MM cell lines and primary specimens and had a robust antitumor effect in a disseminated MM mouse model. Several nucleoside analogs are effective in treating leukemias and T cell lymphomas, and 6-ETI may fill this niche for the treatment of PEL, plasmablastic lymphoma, MM, and other ADK-expressing cancers.
\end{abstract}

\section{Introduction}

The $\gamma$-herpesvirus KSHV, also called HHV-8, is the etiological agent of Kaposi's sarcoma (KS) (1), multicentric Castleman's disease (2), and primary effusion lymphoma (PEL) (3). KS, the most common malignancy in AIDS patients, is a tumor of endothelial origin, while PEL is a rare non-Hodgkin B cell lymphoma that commonly manifests as lymphomatous effusions. Combined antiretroviral therapy has been shown to be effective in some cases of $\mathrm{KS}$, as are other noncurative approaches such as radiation, surgery, and chemotherapy. However, PEL itself remains by and large a highly aggressive and intractable disease, with rapid progression to death. Therefore, the need for specific and effective therapeutics for diseases involving KSHV is pressing, albeit challenging because of the latent nature of the virus.

Two broad approaches can be envisioned to identify new strategies for the treatment of virus-associated malignancies to target specific vulnerabilities conferred by viral infection. The first one is to use existing agents that target pathways that are activated by the virus and are essential for tumor cell survival. The advantage

Authorship note: U. Nayar and J. Sadek contributed equally to this work. Conflict of interest: U. Nayar, J. Sadek, J.D. Warren, and E. Cesarman are inventors on patent PCT/US17/27590, which has been filed for 6-ETI and related structures. Submitted: July 27, 2015; Accepted: March 16, 2017.

Reference information: J Clin Invest. 2017;127(6):2066-2080

https://doi.org/10.1172/JCl83936. of this strategy is the availability of compounds previously tested for other diseases and in clinical use, with documented pharmacological properties. We and other groups have studied the effectiveness of such commercially available or clinically established pathway inhibitors, such as the NF- $\kappa B$ inhibitor Bay11-7082 and the Hsp90 inhibitor PU-H71, in PEL cell lines (4-6). However, none of these have been completely effective in mouse models or involved the use of compounds that are already past early phases of clinical trials. A second approach is to identify specific inhibitors of viral proteins, which provides the unique opportunity for very specific and potentially nontoxic therapy. One possible candidate viral oncogene is vFLIP, a viral latent protein encoded by KSHV and expressed in all infected tumor cells. vFLIP is essential for PEL cell survival as demonstrated by RNAi (7); this effect is mediated by binding of vFLIP to IKK $\gamma$ and activation of the downstream NF- $\mathrm{BB}$ pathway $(8,9)$. We sought to exploit the dependence of PEL cells on vFLIP-induced NF- $\mathrm{B}$ signaling by conducting a high-throughput screen using an NF- $\kappa \mathrm{B}$ reporter cell line, and searching for molecules that inhibit NF- ${ }_{-1} \mathrm{~B}$ in PEL but not in $\mathrm{KSHV}^{-}$cell lines.

We successfully identified a novel inhibitor, 6-ethylthioinosine (6-ETI), a compound that is highly selective for PEL cell lines in vitro and highly efficacious in vivo. Unexpectedly, this compound did not decrease levels of vFLIP or other viral proteins, and toxicity at low concentrations occurred irrespective of vFLIP-induced NF- $\kappa \mathrm{B}$ signaling suppression. Unbiased analysis of mechanisms of resistance using a genomic approach revealed 
A

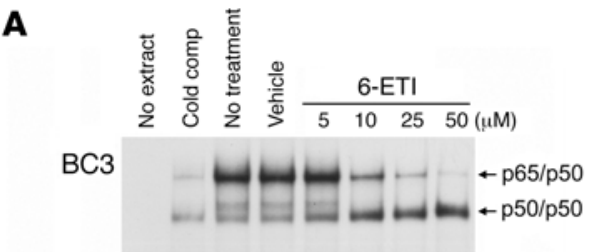

LCL9001

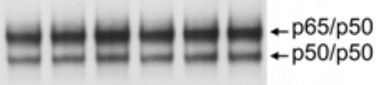

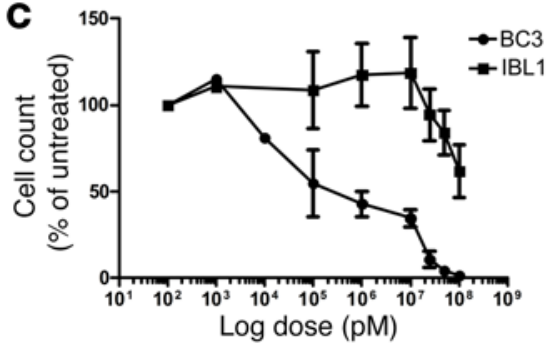

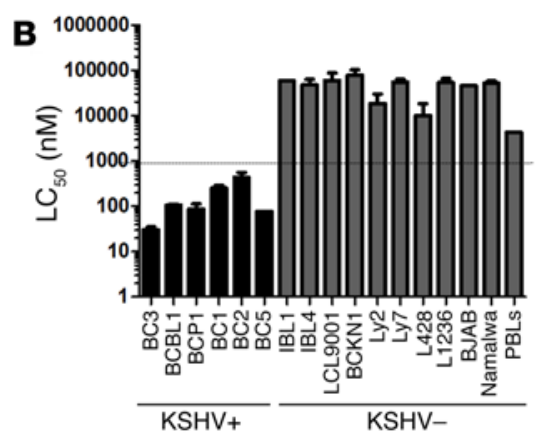
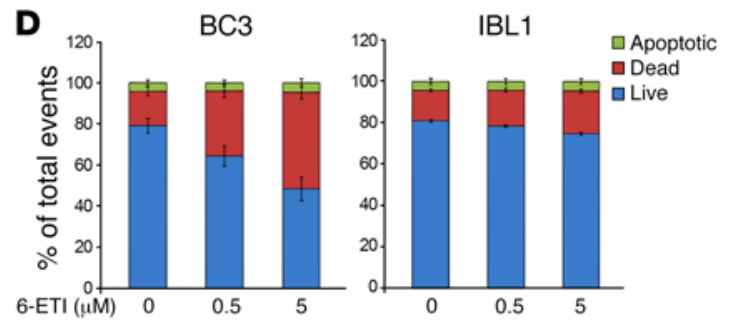

Figure 1. Selection of 6-ETI. (A) BC3 (KSHV $)$ and LCL9001 (KSHV $)$ cells were treated for 24 hours with selected doses of NSC39368 (6-ETI), followed by nuclear extraction and EMSA to determine binding to an NF-KB response element. Control lanes include no compound, competition with cold probe, untreated, and DMSO-treated samples. Experiment was performed 3 separate times. A representative blot is shown. (B) A panel of $\mathrm{KSHV}^{+}$(black bars) and $\mathrm{KSHV}^{-}$(gray bars) cell lines was plated at $1 \times 10^{5}$ cells $/ \mathrm{ml}$ in RPMI with $20 \%$ FBS with increasing concentrations of 6-ETI, followed by analysis for ATP content by CellTiter-Clo assay at 48 hours. LC $_{50}$ s were determined by online EC $_{50}$ software. Results are mean \pm SEM of at least 3 independent experiments. (C) BC3 and IBL 1 cells plated at $1 \times 10^{5}$ cells/ml in RPMI with $20 \%$ FBS at a range of concentrations of 6-ETI were examined for cell death by trypan blue staining-aided cell counts at 48 hours after treatment. Results plotted are mean $\pm \mathrm{SEM}$ of at least 3 independent experiments. (D) BC3 and IBL1 cells plated at $2 \times 10^{5}$ cells $/ \mathrm{ml}$ in RPMI with $20 \%$ FBS were treated with DMSO or $500 \mathrm{nM}$ or $5 \mu \mathrm{M} 6-\mathrm{ETI}$ for 48 hours and analyzed by flow cytometry for cell death after staining with annexin $V$ and 7 -aminoactinomycin D. Results are the average of at least 2 independent experiments (mean \pm SEM).

an unpredicted dependency of 6-ETI on phosphorylation by endogenous adenosine kinase (ADK) for its exquisite selectivity. Furthermore, ADK overexpression was found to serve as a new biomarker for 6-ETI sensitivity in other related tumors, and in particular in multiple myeloma, expanding the scope of this inhibitor as an effective preclinical agent. Our results demonstrate the use of unbiased genomic analysis to identify mechanisms of resistance and of action and to enable target identification of novel lead compounds.

\section{Results}

Development of a high-throughput screening assay using a PELspecific NF- $\kappa B$ reporter cell line. The PEL cell line BC3 was previously modified through transduction of NF- $\mathrm{kB}$-firefly luciferase plasmid, followed by selection and subcloning, to yield the BC3NFkB-luc\#6 single reporter cell line (6). We screened the NIH Training Set (230 compounds) using this cell line, and determined optimal assay conditions for high-throughput screening (Supplemental Table 1; supplemental material available online with this article; https://doi.org/10.1172/JCI83936DS1). The established cell-based luciferase reporter assay was used to screen the NIH Diversity Set (1,981 compounds) at $5 \mu \mathrm{M}$, yielding 60 primary hits that demonstrated at least $50 \% \mathrm{NF}-\kappa \mathrm{B}-$ luciferase inhibition, for an initial hit rate of $3 \%$. Retesting of these hit compounds determined 50 to be genuine.
As a next step to determine specificity, we developed a secondary assay, wherein the single reporter cell line was lentivirally transduced with a constitutive renilla luciferase construct. The transduced cell line was subcloned to generate the double reporter cell line BC3NFRen-luc\#3. We tested the 50 hit compounds using the double reporter cell line, as well as an additional control cell line, U251-pGL3, derived from a glioma cell line, U251, which expresses firefly luciferase constitutively under the control of the SV40 promoter (provided by G. Mellilo, National Cancer Institute). Screening the double reporter and control cell line led to the identification of 3 hit compounds that were found to possess specific antiNF- $\mathrm{KB}$ activity against PEL cells (Supplemental Table 2). We performed detailed chemoinformatic analysis on these hit compounds and identified 66 additional analogs in the NIH compound repository, which were screened by the double reporter assay for structure-activity relationship. Results from this analysis informed further analog identification; 39 additional analogs were screened by the double reporter assay in this second-tier analysis. Rescreening validated 3 analogs of 2 of the parent compounds as additional hits for NF- $\kappa B$ inhibition in PEL cells (Supplemental Table 2).

Selection of 6-ETI. We examined the 6 hit compounds obtained at the end of high-throughput screening (Supplemental Table 2) for inhibition of the NF- $\mathrm{KB}$ pathway in parental BC3 cells. Supplemental Figure 1A shows that the compound NSC39368 effectively inhibited active heterodimer $\mathrm{p} 65 / \mathrm{p} 50$ binding to the NF- $\kappa B$ response element by EMSA. This inhibition occurred in the BC3 PEL cell line, but not in a control lymphoblastoid cell line (LCL9001) containing EBV (Figure 1A). This result enabled us to identify NSC39368 or 6-ethylthioinosine, a nucleoside analog, hereafter referred to as 6-ETI, as the most promising compound for further investigation.

$\mathrm{NF}-\mathrm{kB}$ reporter assays performed on BC3NFRen-luc\#3 cells confirmed that 6-ETI inhibited NF- $\mathrm{kB}$ activity in this cell line as early as 6 hours after treatment (Supplemental Figure 1B). 6-ETI treatment in BC3 cells was found to decrease levels of active subunit p65 in the nucleus, while leaving inactive subunit p50 unchanged (Supplemental Figure 1C), and it also decreased levels of IL-6, an NF-kB-dependent gene in PEL cells (Supplemental Figure 1D). Other components of classical NF- $\mathrm{kB}$ signaling, including 
A

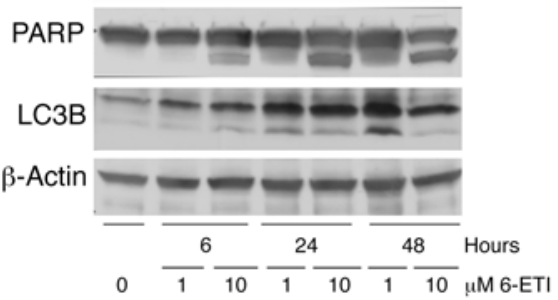

C

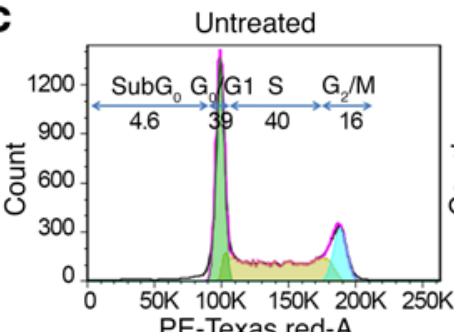

E

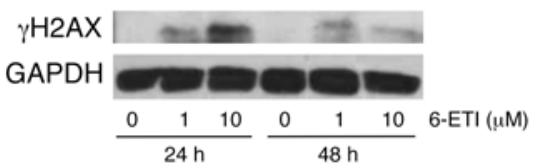

B

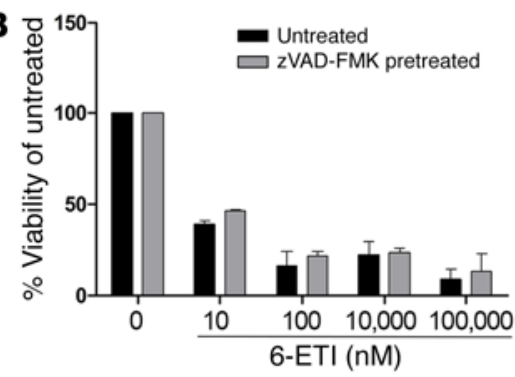

D

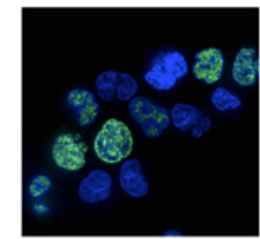

Untreated

$\mathbf{F}$

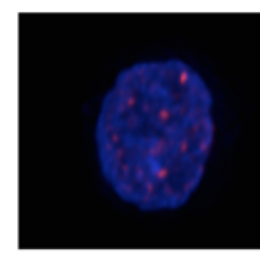

Untreated

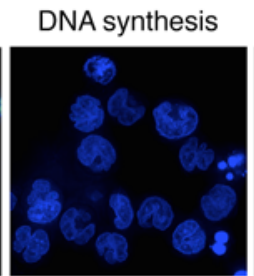

$1 \mu \mathrm{M} 6-\mathrm{ETI}$

$\gamma \mathrm{H} 2 \mathrm{AX}$

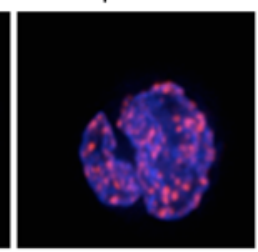

$10 \mu \mathrm{M} 6-\mathrm{ETI}$

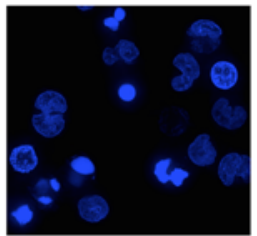

$10 \mu \mathrm{M} 6-\mathrm{ETI}$

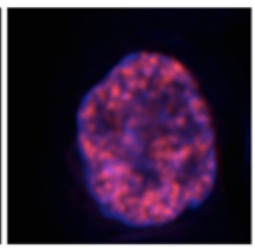

UV irradiated

Figure 2. Mechanism of 6-ETI-induced cell death. (A) BC3 cells treated with the indicated doses of 6-ETI at the shown time points were analyzed by Western blotting on whole cell extracts for PARP cleavage as an indicator of apoptotic induction and for LC3B cleavage as an indicator of autophagosome formation. Actin was used as a loading control, and results shown are representative of at least 3 independent experiments. (B) BC3 cells were pretreated or not with $80 \mu \mathrm{M}$ zVAD-FMK, a pan-caspase inhibitor, to inhibit apoptosis. After 3 hours, cells were treated at the indicated doses of 6-ETI. Cells lysed at 48 hours were examined by CellTiter-Glo to measure viability, with results normalized to untreated cells at each condition. The mean \pm SEM of 3 independent experiments is shown. (C) BC3 cells treated with DMSO or $1 \mu \mathrm{M} 6$-ETI for 24 hours were stained with propidium iodide, followed by flow-based cell cycle analysis. The obtained histograms were modeled by Flowjo. Results shown are representative of at least 3 independent experiments. (D) BC 3 cells were treated with DMSO or $1 \mu \mathrm{M}$ or $10 \mu \mathrm{M}$ 6-ETI for 24 hours, then labeled with $10 \mu \mathrm{M}$ EdU for 2 hours. EdU incorporation into the newly synthesized DNA was visualized by staining of cells using Click-iT EdU Alexa Fluor 488 cocktail, and the nucleus was counterstained using Hoechst. Immunofluorescence images are representative of 2 independent experiments. (E) Immunoblotting for $\gamma \mathrm{H} 2 \mathrm{AX}$ is shown after treatment with 6-ETI at the indicated time points and doses. Result shown is representative of 3 independent experiments. (F) Representative immunofluorescence images of 2 independent experiments showing nuclear localization of $\gamma \mathrm{H} 2 \mathrm{AX}$ after treatment with 6-ETI for 48 hours. As a positive control, BC3 cells were treated with UV radiation to induce DNA damage. See related Supplemental Figure 2 for BC1 data. Original magnification, $\times 100$.

IKK $\alpha$, IKK $\beta$, and I $\mathrm{B} \alpha$, were not consistently affected by 6-ETI treatment (Supplemental Figure 1D). Thus, like other inhibitors of NF- $\kappa \mathrm{B}(10,11), 6$-ETI inhibited p65 nuclear accumulation, but the mechanism for this selective inhibition of $\mathrm{p} 65$ nuclear localization in PEL was not evident from immunoblot analysis of known upstream regulators of this process.

6-ETI specifically inhibits PEL cell viability. To assess toxicity and confirm selectivity toward PEL, we examined 6-ETI for its effectiveness against a panel of cell lines, including $\mathrm{KSHV}^{+} \mathrm{PEL}, \mathrm{KSHV}^{+} \mathrm{EBV}^{+}$ $\mathrm{PEL}, \mathrm{EBV}^{+}$immunoblastic lymphomas (IBL1), EBV ${ }^{+}$lymphoblastoid cell lines (LCL), EBV ${ }^{-}$Hodgkin's lymphoma (HL) cell lines, $\mathrm{EBV}^{+}$and $\mathrm{EBV}^{-}$Burkitt's lymphoma (BL) cell lines, virus-negative diffuse large B cell lymphoma (DLBCL) cell lines, several of which are known to be NF- $\kappa \mathrm{B}$-dependent, and normal peripheral blood leukocytes. We used an ATP-based luminescent cell viability assay (CellTiter-Glo) to determine median lethal concentrations $\left(\mathrm{LC}_{50} \mathrm{~s}\right)$ at 48 hours (Figure 1B) after treatment initiation, and observed a striking correlation of effectiveness with KSHV infection status of the cell. Cell lines that were $\mathrm{KSHV}^{+}$had $\mathrm{LC}_{50}$ s in the mid-nanomolar range, varying from approximately 30 to $435 \mathrm{nM}$, while all $\mathrm{KSHV}^{-}$ cell lines tested, regardless of tumor type, EBV infection status, or $\mathrm{NF}-\kappa \mathrm{B}$ dependence, exhibited $\mathrm{LC}_{50} \mathrm{~s}$ in the micromolar $(10-80 \mu \mathrm{M})$ range, showing an average fold difference in sensitivity of approximately 275 ( $P=0.0001$, unpaired $t$ test). To ensure concordance between ATP levels and cell death, viability for a selected PEL and non-PEL cell line was also assessed by trypan blue exclusion, yielding $\mathrm{LC}_{50}$ s of $74 \mathrm{nM}$ and $134 \mu \mathrm{M}$, respectively (Figure 1C). The 6-ETItreated PEL cell line also had a higher proportion of dead cells as determined by annexin V/7-aminoactinomycin D staining (Figure 1D). Notably, the 6-ETI doses that had been found to be effective in inhibiting NF- $\mathrm{B}$ in PELs were in the range of $10-50 \mu \mathrm{M}$, while cell viability was impacted at nanomolar doses, indicating that NF- $\mathrm{BB}$ inhibition was a secondary effect of this compound and not the primary mechanism involved in cell death. 
A

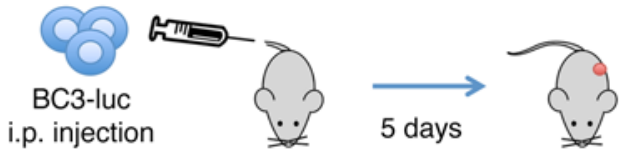

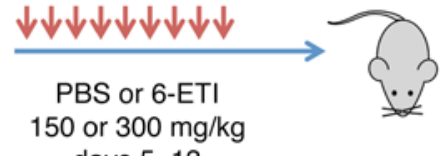

days $5-13$

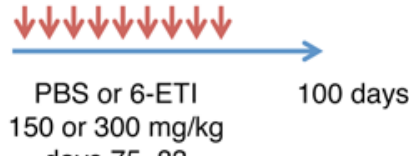

days $75-83$
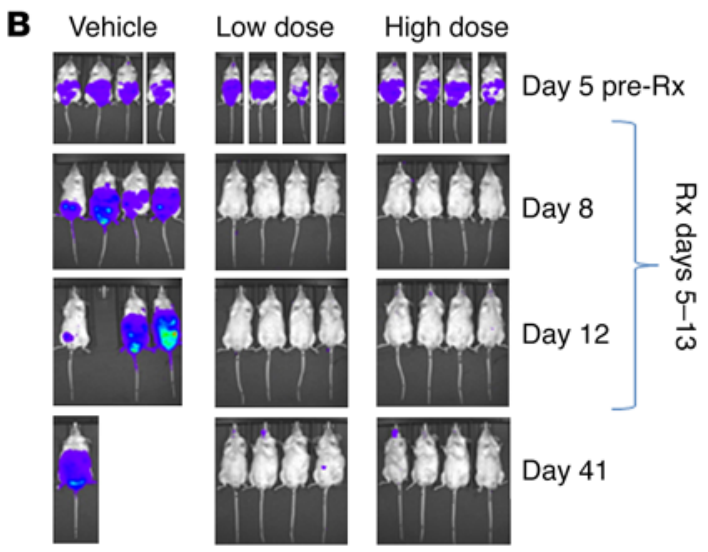

C

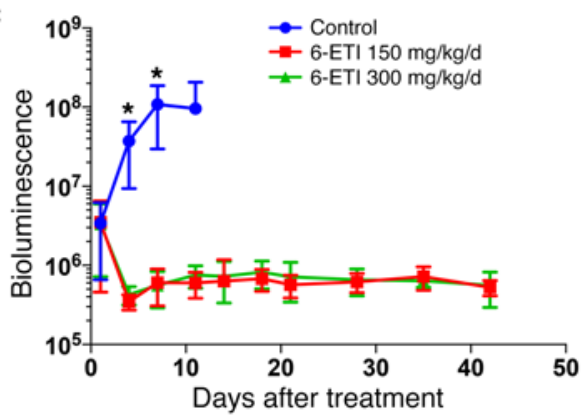

D

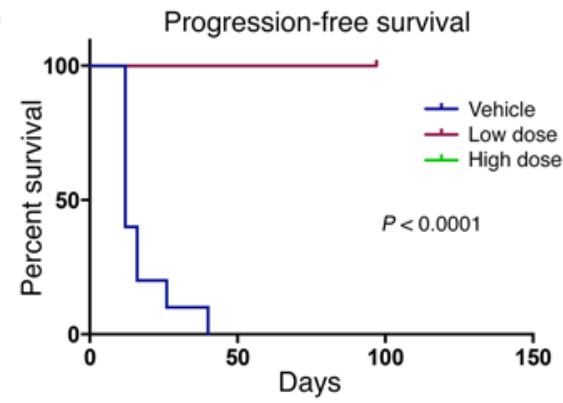

7im 190

Day 75 pre-Rx

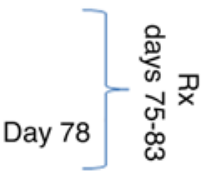

E

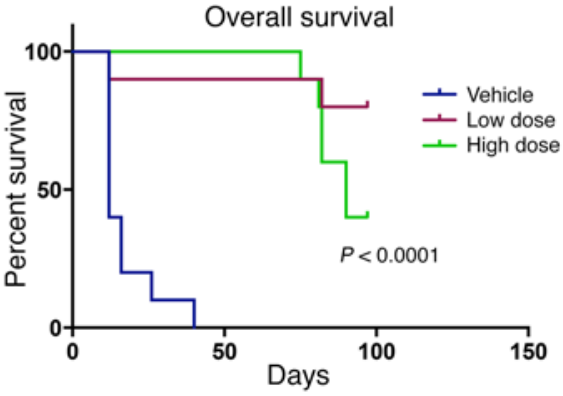

Figure 3. 6-ETI is effective in vivo in a PEL xenograft mouse model. (A) Scheme of BC3-luc tumor cell engraftment in NOD/SCID mice and treatment with 6-ETI. (B) NOD/SCID mice randomized into vehicle $(n=10)$, low-dose $(150 \mathrm{mg} / \mathrm{kg} / \mathrm{d})(n=10)$, and high-dose $(300 \mathrm{mg} / \mathrm{kg} / \mathrm{d})(n=10)$ treatment groups after BC3NFRen-luc\#3 tumor engraftment were followed by weighing and luciferase imaging in vivo to follow tumor progression. Mice in both treatment groups were treated for 2 separate 9-day intervals as indicated. Representative results from the first trial of 2 are shown. (C) Quantitation of tumor burden by bioluminescence imaging from 2 independent trials. Data are shown through day 41. Statistical analysis was performed using 1-way ANOVA ( $P=0.0032$ at day 11 after treatment). Statistical analysis comparing vehicle- and low and high dose-treated mice separately at specific time points was also performed using unpaired $t$ test $\left({ }^{*} P \leq 0.05\right)$. ( $\mathbf{D}$ and $\left.\mathbf{E}\right)$ Kaplan-Meier analysis of progression-free survival $(n=22)(\mathbf{D})$ and overall survival $(n=30)(\mathbf{E})$ was performed on all mice from both trials, and the results are color-coded by treatment group. In the progression-free survival curve (D), the low-dose ( $n=8)$ and high-dose $(n=4)$ treatment arms showed complete overlap, as by day 100 all live mice were tumor free, and none had died of tumor. The difference in survival curves was analyzed by log-rank (Mantel-Cox) test $(P<0.0001)$.

PEL cells undergo apoptosis and necrosis upon 6-ETI treatment. In order to determine the mechanism of 6-ETI-induced cell death, we examined treated cells for activation of classical cell death pathways, including apoptosis, autophagy, and necrosis. We tested the effect of treatment of 2 PEL cell lines with $1 \mu \mathrm{M}$ 6-ETI, as this concentration is close to the $\mathrm{LC}_{95}$, which allowed us to capture the full effect of the drug while not having any effect in resistant cell lines. We also tested $10 \mu \mathrm{M}$, which exceeds the effective concentration but provides information on earlier effects and is a dose at which there is no toxicity or any other appreciable effects in resistant cell lines. PARP cleavage was used as a marker of apoptosis in PEL cells treated with 6-ETI. As seen in Figure 2A and Supplemental Figure 2, 6-ETI induced small but observable increases in apoptosis in $\mathrm{BC} 1$ and $\mathrm{BC} 3$ cells at the $1 \mu \mathrm{M}$ dose as early as 24 hours. Because KSHV vFLIP has also been implicated in autophagic cell death, we performed Western blotting to determine the extent of activation of this pathway after 6-ETI treatment in PEL cells. There was some induction of autophagy, as assessed by appear- 
A

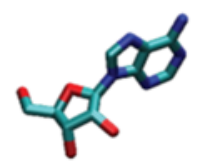

Adenosine
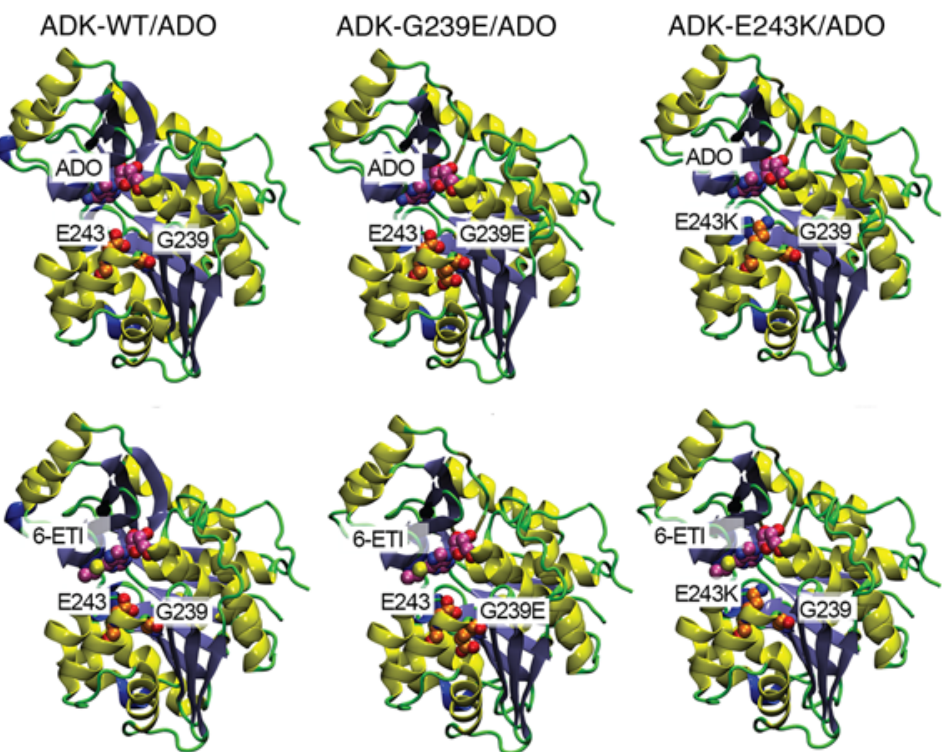

ADK-WT/6-ETI

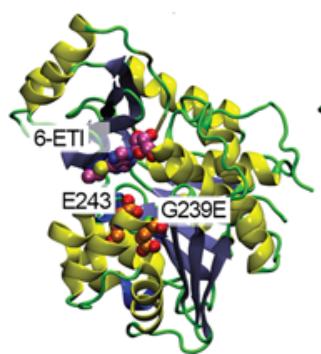

ADK-G239E/6-ETI

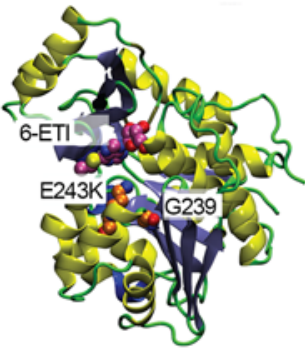

ADK-E243K/6-ETI
B

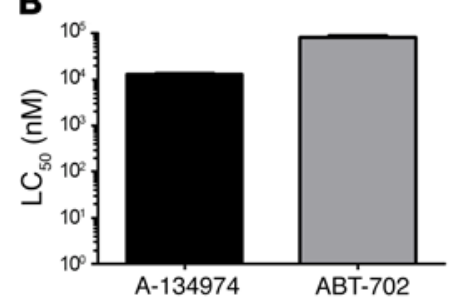

c

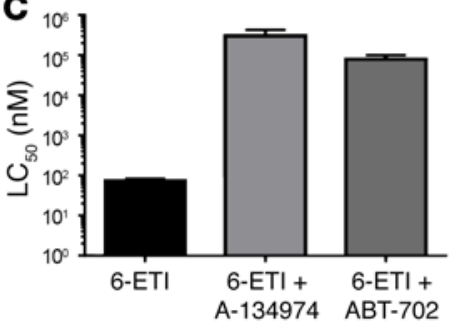

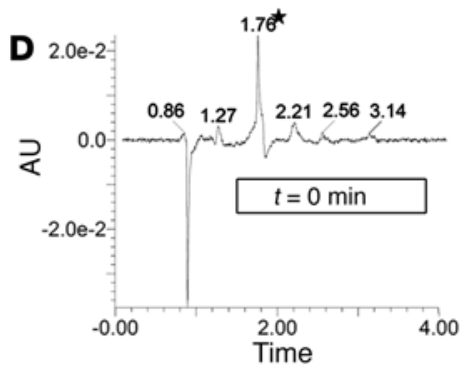

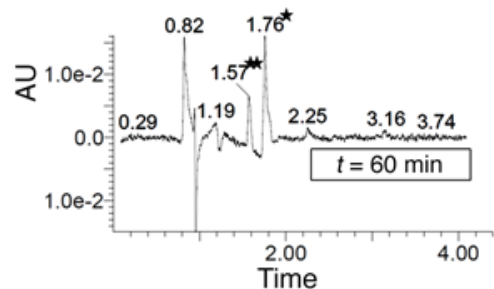

E

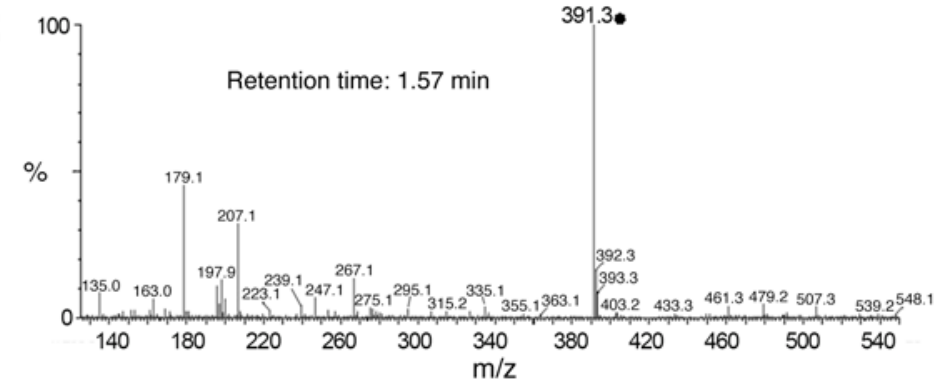

Figure 4. ADK is required for 6-ETI activation. (A) Figure indicates locations of independent recurrent mutations G239E and E243K on ADK, discovered by SNP calling on RNA-Seq data from 6-ETI-resistant clones. Schrödinger's Maestro was used to model ADK binding to adenosine (ADO) (top panel), and postulated binding to 6-ETI (bottom panel). (B) BC3 cells treated at a range of concentrations of A-134974 or ABT-702 were analyzed by CellTiter-Clo assay for viability at 48 hours. Results are shown as the mean $\pm S E M$ of at least 3 independent experiments where $\mathrm{LC}_{50} \mathrm{~s}$ were determined using online $\mathrm{EC}_{50}$ software. (C) 6-ETI viability dose curves were set up on BC3 cells pretreated with $1 \mu \mathrm{M}$ ADK inhibitors A-134974 and ABT-702 or DMSO, followed by treatment with 6-ETI. The fold increases in 6-ETI LC 50 are shown via bar graph, representing the mean \pm SEM of at least 3 independent experiments. (D) ADK kinase assay to test 6-ETI phosphorylation was carried out by mixing of kinase assay components with 6-ETI, followed by acetonitrile quenching at various time points. Reaction mixture at each time point was injected into HPLC to detect the appearance of the phosphorylated peak. Chromatogram for the 6-ETI standard under the HPLC conditions indicates the appearance of a peak at retention time 1.76 minutes, indicated by a star. Chromatograms at $t=0$ and $t=60$ minutes both contain this peak, while $t=60$ reveals the appearance of a secondary peak at 1.57 minutes (indicated by double star). (E) Mass spectrometry analysis was performed on the fraction of the $t=60$ reaction mixture retrieved at 1.57 minutes of HPLC. The MS revealed a prominent peak at 391.3 (indicated by asterisk), corresponding exactly to the expected molecular weight of phospho-6-ETI.

ance of the LC3B cleavage product, apparent at the $1 \mu \mathrm{M}$ dose at the 24 hour time point (Figure 2A and Supplemental Figure 2).

Cells with low ATP levels have been observed to preferentially undergo necrosis over apoptosis (12). Since 6-ETI-treated cells have low ATP content upon treatment as determined by CellTiterGlo assay, we examined PEL cells for the appearance of necrosis, as determined by caspase-independent cell death. In order to do this, we pretreated PEL cells with the pan-caspase inhibitor zVADFMK, followed by 6-ETI treatment at selected doses (Figure 2B). PEL cells pretreated with zVAD-FMK still underwent cell death upon 6-ETI treatment compared with untreated cells. This confirmed that 6-ETI-induced cell death is largely caspase-indepen- 
A

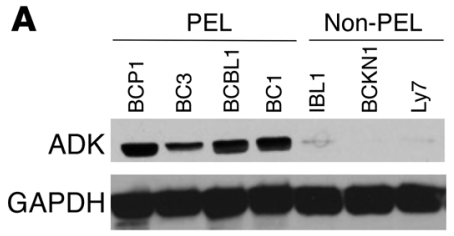

B

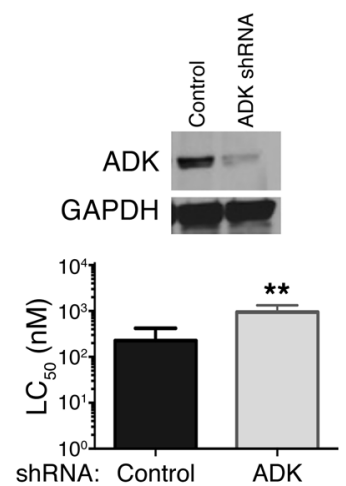

C

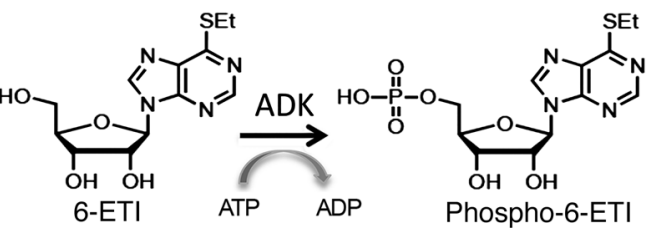

D

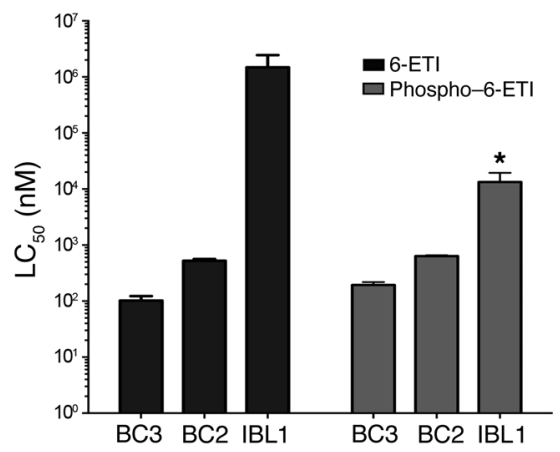

Figure 5. ADK levels determine sensitivity to 6-ETI. (A) A panel of $\mathrm{KSHV}^{+}$and $\mathrm{KSHV}^{-}$lymphoma cell lines were plated at viability assay conditions (i.e., $1 \times 10^{5}$ cells $/ \mathrm{ml}$ in RPMI with $20 \% \mathrm{FBS}$ ), and whole cell extracts obtained at 24 hours were examined for ADK levels by Western blotting. GAPDH was used as a loading control. Results shown are representative of more than 3 independent experiments. (B) Successful lentiviral shRNA-mediated knockdown of ADK in the pancreatic adenocarcinoma cell line PATu-8988T was examined by Western blot using an ADK antibody. A representative blot of 3 independent experiments is shown. 6-ETI viability assays at 24 hours were performed on the knockdown cell line compared with control. $\mathrm{LC}_{50}$ graphed is the average of 5 independent experiments (mean $\pm \mathrm{SEM}$ ). Statistical analysis was performed using Mann-Whitney unpaired test $\left(P=0.0079,{ }^{* *} P \leq 0.05\right)$. (C) ADK normally phosphorylates adenosine to convert it into adenosine monophosphate (AMP) and similarly adds a phosphate from ATP into 6-ETI to convert it into p6-ETI. (D) PEL cell lines BC3 and BC2 and DLBCL cell line IBL1 were treated with increasing concentrations of 6-ETI and phospho-6-ETI for 48 hours. Results plotted are the average of 4 independent experiments (mean \pm SEM). Statistical analysis was performed using Mann-Whitney unpaired test $\left(P=0.0317,{ }^{*} P \leq 0.05\right)$.

dent, and that necrosis is a major pathway of cell death induced by this compound, although apoptosis and autophagy also occur, at least in a subset of cells and under some experimental conditions.

6-ETI induces $S$ phase arrest. We examined effects on the cell cycle upon 6-ETI treatment by propidium iodide flow cytometry. We observed an increase in the fraction of cells in the sub- $G_{0}$ phase upon 6-ETI treatment, likely reflective of some apoptosis, but we also detected a reproducible increase in the percentage of cells in S phase 24 hours after treatment in $\mathrm{BC} 3$ cells. This represents an induction of $\mathrm{S}$ phase arrest by the compound, as modeled by FlowJo (Figure 2C). This accumulation of cells in the $\mathrm{S}$ phase is consistent with incorporation of 6-ETI into DNA during DNA synthesis, thereby blocking extension and causing stalling of replication forks. We investigated the effect of 6-ETI on DNA synthesis by using a Click-iT 5-ethynyl-2'-deoxyuridine (EdU) assay, which showed a complete inhibition of DNA synthesis upon $1 \mu \mathrm{M}$ treatment of 6-ETI for 24 hours (Figure 2D). Similarly, we also observed reduction in BrdU incorporation upon exposure to 6-ETI treatment (data not shown). This process leads to the formation of double-stranded breaks in DNA, thus triggering a DNA damage response, a phenomenon that has been observed with other nucleoside analogs (13). To determine whether 6-ETI similarly triggers a DNA damage response, we assessed 6-ETI-treated PEL cells for the presence of $\gamma \mathrm{H} 2 \mathrm{AX}$, a well-known marker of double-stranded breaks. Immunoblot analysis showed the appearance of $\gamma \mathrm{H} 2 \mathrm{AX}$ at 24 hours after treatment with $1 \mu \mathrm{M}$ and $10 \mu \mathrm{M}$ doses of 6-ETI (Figure 2E and Supplemental Figure 2). Nuclear accumulation of $\gamma \mathrm{H} 2 \mathrm{AX}$ was also seen by immunofluorescence (Figure 2F), confirming that 6-ETI induces DNA damage.

6-ETI does not inhibit viral latent protein levels. Since several viral latent proteins have been shown to directly modify important cellular signaling pathways, such as vFLIP's critical role in sustained NF-אB induction in PELs (7), and since 6-ETI was isolated using a PEL-specific NF-KB reporter assay, we postulated that this compound may exert its effects on PEL cell viability and NF- $\mathrm{KB}$ signaling through inhibition of vFLIP or other oncogenic viral gene products. Accordingly, we examined the levels of the major KSHV latent proteins upon 6-ETI treatment by immunoblotting. However, no reduction was seen in latent viral protein expression (Supplemental Figure 3A). This unexpected effect was also observed when we examined treated cells for levels of these transcripts by quantitative reverse transcription PCR at 24 hours (Supplemental Figure 3B).

Since lytic reactivation triggered by the compound could be a mechanism of cell death, we examined 6-ETI's effects on viral lytic gene expression. Analysis of immediate-early (ORF50) and late lytic transcripts (K8.1) by quantitative reverse transcription PCR demonstrated a minor induction of immediate-early and mild suppression of late transcripts at the highest dose (Supplemental Figure 3B). The immediate-early gene viral IL-6 (vIL-6), which has also been found to be expressed at low levels during latency (14), was increased in expression at higher doses, paralleling the other latent genes. To further investigate the compound effects on the lytic viral cycle, we used the JSC1 PEL cell line infected with recombinant KSHV (rKSHV.219), which expresses GFP under the control of the EF1- $\alpha$ promoter in all cells and red fluorescent protein (RFP) under the control of the early lytic PAN promoter of KSHV (15). This cell line thus expresses GFP constitutively, and RFP only when the lytic cycle is activated. As Supplemental Figure 3C shows, there was an induction of lytic reactivation by 6-ETI compared with the lytic phase inducer 12-O-tetradecanoylphorbol-13-acetate (TPA) in a small proportion of cells, which increased at higher doses and longer time points. This induction of lytic reactivation does not explain the cell death that occurs in a larger proportion of PEL cells with toxic doses of 6-ETI.

6-ETI is highly effective in vivo. We tested 6-ETI using a traceable reporter xenograft model of PEL (6). We treated cohorts of 10 mice with vehicle alone or 150 or $300 \mathrm{mg} / \mathrm{kg} / \mathrm{d}$ 6-ETI for 9 days (Figure 3A). These doses were selected based on NIH in vivo anti- 
A

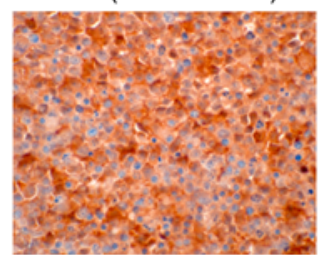

Tonsil

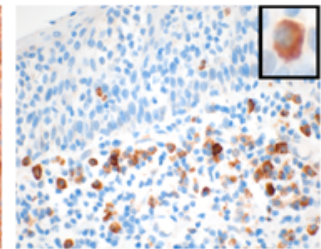

PEL

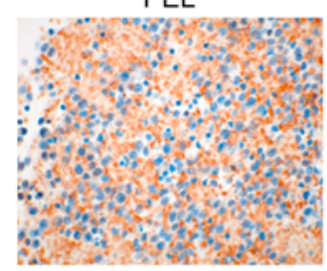

B
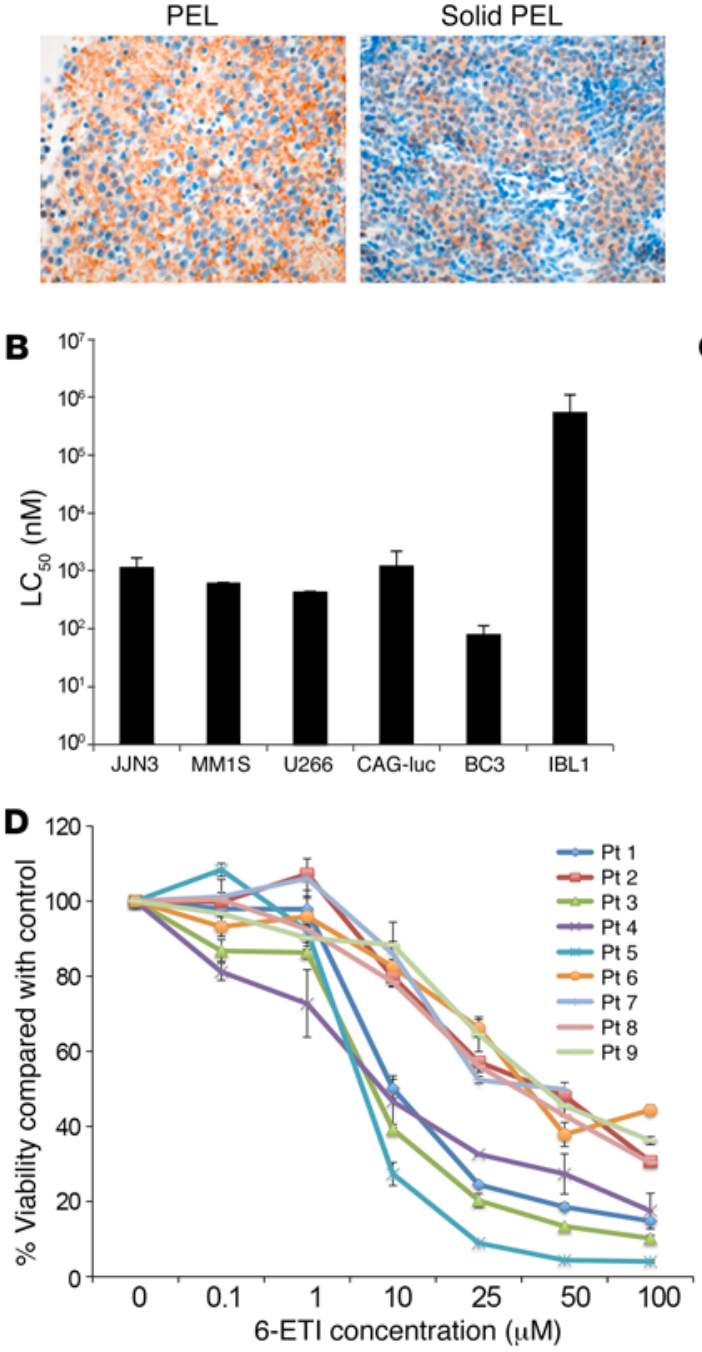

Tonsil plasma cells

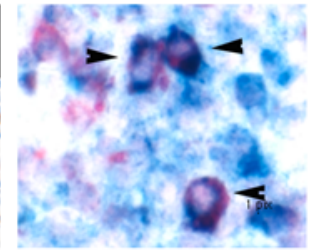

CD138-blue

ADK-red
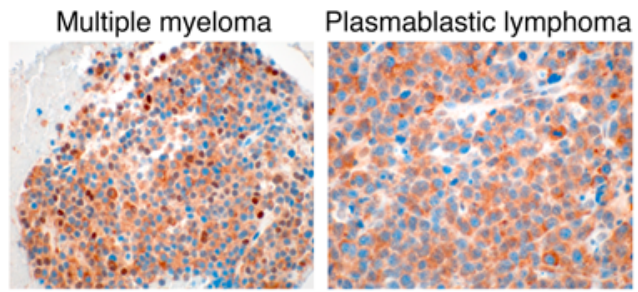

C

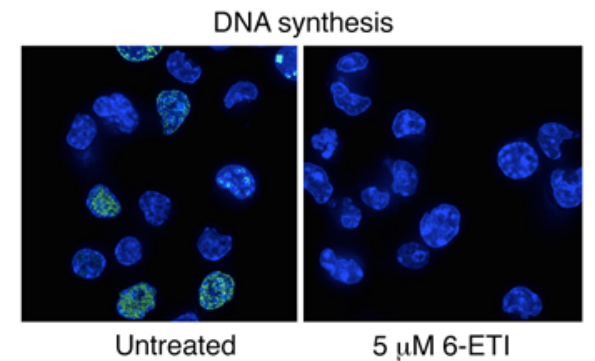

E

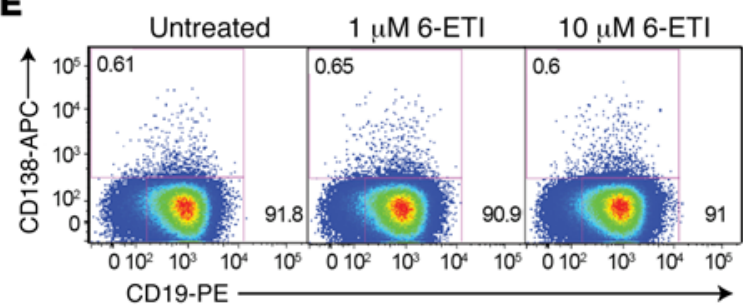

Figure 6. Expression of ADK and sensitivity to 6-ETI in plasma cell tumors. (A) BC3 cells' ADK expression was evaluated by immunohistochemistry in the BC3 cell line, hyperplastic tonsils, and PEL, MM, and plasmablastic lymphoma primary tumors (original magnification, $\times 60$ ). Inset: In the image of a tonsil section, a positive cell with morphological features of a plasma cell is enlarged. Two-color immunohistochemistry showing a high-power image of plasma cells expressing both ADK and CD138. (B) LC 5 s for MM cell lines treated with 6-ETI for 48 hours were determined by CellTiter-Clo assay. BC3 was used as a positive control and IBL1 as a negative control for drug sensitivity. Shown is the mean \pm SEM of 2 independent experiments, where each condition was performed in duplicate in each experiment. (C) U266 cells were treated with DMSO or $5 \mu \mathrm{M} 6$-ETI for 24 hours, then labeled with $10 \mu \mathrm{M}$ EdU for 2 hours. EdU incorporation into the newly synthesized DNA was visualized using Click-iT EdU assay. The immunofluorescence images are representative of 2 independent experiments. Original magnification, $\times 100$. (D) MM patient specimens $(n=9)$ were treated with increasing concentrations of 6 -ETI ex vivo, followed by analysis for ATP content by CellTiter-Clo assay at 24 hours. $\mathrm{LC}_{50}$ s were determined using GraphPad Prism. (E) Flow cytometry analysis showing the number of normal plasma cells isolated from human tonsils $\left(\mathrm{CD}^{-} 9^{-}, \mathrm{CD} 138^{+}\right)$before and after 6-ETI treatment. Data are representative of 2 independent experiments.

cancer drug screens with this drug, which used $200 \mathrm{mg} / \mathrm{kg}$ for 9 days with no reported toxicities. Luciferase imaging in vivo clearly showed a striking and immediate regression of the implanted xenograft within 3 days of treatment, which began at day 5 after engraftment (Figure 3B). Quantification of the luminescent sig- nal as a measure of tumor burden is shown in Figure 3C. In 2 mice treated at the lower dose level $(150 \mathrm{mg} / \mathrm{kg} / \mathrm{d})$, tumor recurred 2 months after cessation of treatment, but responded again upon retreatment, documenting an impressive response in cases of both incipient and well-established tumors. Kaplan-Meier analysis of 
A

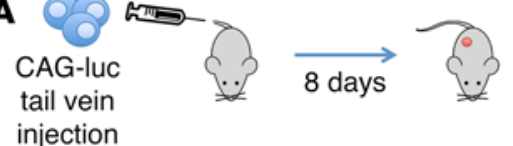

B

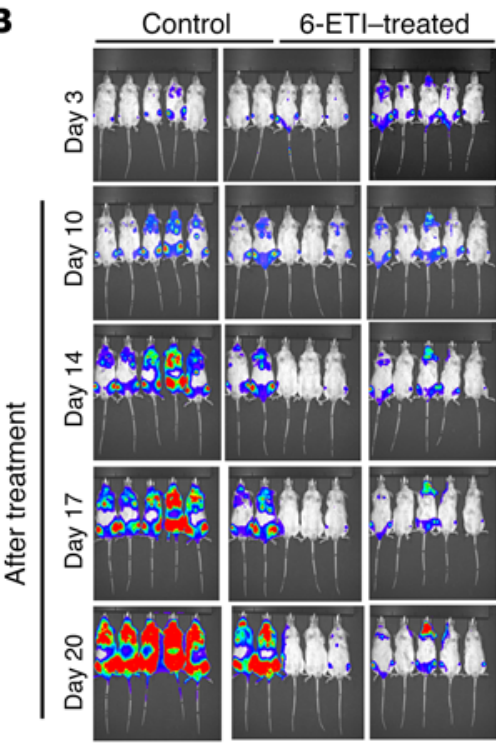

$\downarrow \downarrow \downarrow \downarrow \downarrow \downarrow \downarrow \downarrow \downarrow \downarrow$

PBS or $6-E T I 200 \mathrm{mg} / \mathrm{kg} \quad 20$ days daily $\times 9$ days

C

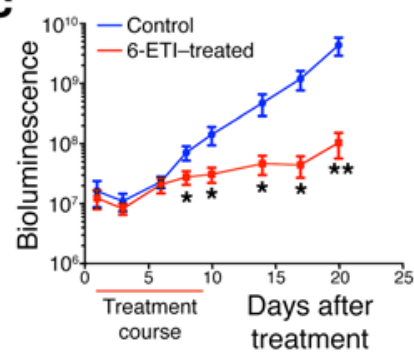

D

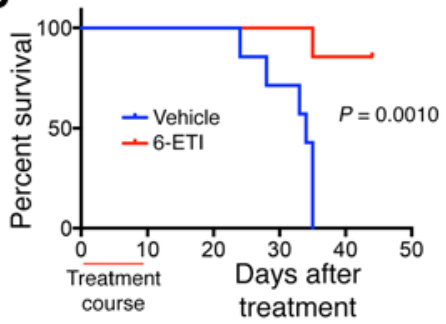

Figure 7. 6-ETI inhibits tumor growth in a disseminated MM mouse model. (A) A schematic of engraftment of CAG-luc MM cell line into NOD/SCID mice and treatment with 6-ETI. (B) When tumors engrafted (at day 8), mice were injected i.p. with vehicle (7 mice on the left) or 6-ETI (8 mice on the right) for 9 days at $200 \mathrm{mg} / \mathrm{kg} / \mathrm{d}$, and tumor progression was monitored using in vivo luciferase imaging. (C) Bioluminescence representing tumor burden after 20 days of follow-up in the vehicle-treated (blue line) versus 6-ETI-treated group (red line) is indicated. Statistical analysis was performed using unpaired $t$ test ${ }^{* *} P=0.0076$ at day 20 after treatment and $P<0.05$ at other time points indicated by ${ }^{*}$ ). (D) Kaplan-Meier survival analysis was performed on all mice, and the results are color-coded by treatment group. The difference in survival curves was analyzed by log-rank (MantelCox) test $(P=0.0010)$. the trials indicated a startling protective effect of 6-ETI treatment on the length of progression-free survival in mice (Figure 3D; $P<$ 0.0001), with the study being terminated at the 100-day mark. There were no obvious toxicities from the treatment, although a few long-surviving mice in the treatment groups succumbed to unrelated infections at the site of repeated luciferase injection. Nevertheless, both low-dose- and high-dose-treated mice had a significant prolonged overall survival compared with untreated mice, as shown in Figure 3E.

Mechanism of selectivity revealed by unbiased genomic analysis. In order to determine the primary target of 6-ETI at effective doses, we performed unbiased genomic sequencing of resistant clones obtained from the parental PEL cell line BC3 through selection. We used transcriptome sequencing for this analysis, in order to simultaneously obtain gene sequences and expression levels. To produce resistant clones, $\mathrm{BC} 3$ cells were grown in 96-well plates at escalating doses of compound up to $10 \mu \mathrm{M}$ over the course of several months, and the resultant clones were tested by viability assay for sensitivity to the compound. We successfully obtained 6 independent clones arising from different wells of 2 plates, NSCE-1, NSCE-2, NSCE-3, NSCE-6, NSCE-7, and NSCE-8, that were fully resistant to 6-ETI (Supplemental Figure 4). Additionally, we were able to culture ex vivo tumor cells from 2 mice in the low-dose cohort of the xenograft trial that developed late recurrent tumors after 2 rounds of treatment, and place these under selection with 6-ETI to yield completely resistant xenograft outgrowth clones. These cell lines were designated 2-3low and 3-5low (Supplemental Figure 4).

We extracted RNA from resistant clones and duplicate samples of the parental cell line, and performed transcriptome sequencing followed by bioinformatic analysis (51 bp single-end reads, mean library size 58.5 million reads per clone) to identify mutations linked to resistance. We reasoned that recurrently observed mutations across multiple independent clones might provide key infor- mation regarding either the target of the compound or other pathways important for its mechanism of action. Both human and KSHV genomes were used as reference sequences for comparison, in order to enable comprehensive target identification. The only recurrently altered gene identified by single-nucleotide variant analysis of the RNA sequencing data was adenosine kinase ( $A D K)$. We found 2 independent mutations (G239E and E243K) in this gene in 2 unrelated resistant clones (Figure $4 \mathrm{~A}$ and Supplemental Table 3).

6-ETI requires endogenous ADK for activity. ADK is the enzyme that catalyzes the transfer of $\gamma$-phosphate from ATP to adenosine or inosine, forming AMP and ADP in the process. This reaction thus constitutes the first step of de novo purine biosynthesis, and makes ADK an essential enzyme whose knockout is lethal in mice (16). ADK is required to control levels of cellular adenosine, which can be toxic if left unregulated (17), while ADK inhibition serves a host of other protective signaling functions $(18,19)$.

The ADK mutations were modeled based on the x-ray crystallographic structure of human adenosine kinase (1BX4) using Maestro (Schrödinger) (Figure 4A). We found that the mutated residues do not directly interact with the substrate or residues in the substrate-binding site, but are close to it and therefore may conceivably contribute to the observed phenotype by affecting the conformational dynamics of the kinase. We examined this gene in depth across all 7 resistant clones, and found recurrent changes in the form of exon loss sparing the active site in 3 additional cases (Supplemental Table 3 and Supplemental Worksheet 2). In the 2 cases where there were no mutations or active site deletions, there was a significant (10-fold) downregulation of gene expression compared with that in the control cell lines. These results indicated that ADK inactivation confers PEL cell resistance to 6-ETI. Conversely, there were no recurrent missense or nonsense mutations in KSHV genes occurring within the resistant cell lines, or significant changes in viral gene expression. There was no over- 


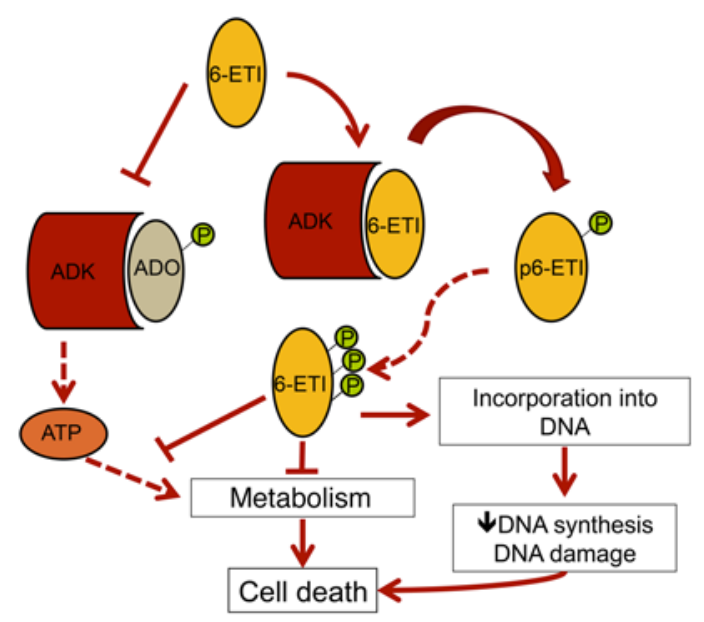

Figure 8. Model for 6-ETI's mechanism of action. 6-ETI competes with adenosine (ADO) and other nucleosides for binding to and phosphorylation by ADK, which inhibits ATP-dependent metabolic processes. This also allows 6-ETI to be phosphorylated and activated by ADK, with subsequent phosphorylation steps that allow the compound to be incorporated into DNA and possibly RNA, leading to DNA synthesis inhibition, DNA damage response, and cell death.

expression of multidrug efflux pumps either, although there were transcriptional changes in a number of genes (complete list provided in Supplemental Worksheet 1).

The inactivation of ADK in every single resistant clone suggests 2 alternative scenarios: (a) that ADK is a direct target of 6-ETI and that ADK is necessary for the survival of PEL cells; or (b) that 6-ETI is a prodrug that is activated by ADK. The latter possibility is supported by previous data indicating that ADK can activate other nucleoside analogs, including several important antiviral compounds such as ribavirin (20-22), triciribine (23), mizoribine (24), and tiazofurin (25), although these compounds can also be activated by a variety of other kinases (26). Such a model was also supported by the locations of the observed mutations, one at and one near the active site of the enzyme, suggesting the involvement of such interactions in drug activity.

In order to differentiate between these 2 scenarios, we first treated BC 3 cells with 2 different ADK inhibitors, A-134974 (27) and ABT-702 (28), and showed that neither of these inhibitors showed toxicity, with $\mathrm{LC}_{50} \mathrm{~s}$ in $\mathrm{BC} 3$ cells of greater than $10 \mu \mathrm{M}$ (Figure 4B), in contrast to their known $\mathrm{IC}_{50}$ of $\mathrm{ADK}$ inhibition in intact cells of $50 \mathrm{nM}$ and $51 \mathrm{nM}$, respectively. To test the alternative model that ADK activates 6-ETI, we performed 6-ETI viability assays in PEL cells that were pretreated with these highly selective ADK inhibitors. We demonstrated through competitive viability curves on $\mathrm{BC} 3$ cells that pretreatment with these inhibitors effectively rescued PEL cells from cell death induced by 6-ETI treatment (Figure 4C).

The mechanism of activation of some nucleoside analogs by ADK involves phosphorylation at the 5 ' end of the "prodrug," converting the molecule into the active "drug" form (29). We examined whether 6-ETI is similarly phosphorylated by ADK using an in vitro kinase assay in which ADK and ATP were mixed with 6-ETI and quenched by acetonitrile at different time points. Samples were injected into an HPLC, followed by mass spectrometry (MS) analysis to enable identification of the putative phospho-6-ETI product. HPLC revealed the appearance of a second peak with a lower retention time (1.57 minutes) compared with the 6-ETI standard (1.76 minutes) as early as 5 minutes after the start of the reaction, and more apparent at 60 minutes (Figure 4D). MS analysis of the 1.57-minute eluent from the 60-minute sample indicated that this new peak contained a primary species of $391.3 \mathrm{Da}$ (Figure $4 \mathrm{E}$ ). According to the $[M-H]$ rule for negative electrospray mode of MS, this corresponds exactly to the expected molecular weight of activated phospho-6-ETI, thus conclusively proving that ADK phosphorylates 6-ETI directly.

Endogenous ADK levels determine sensitivity to 6-ETI. We assessed whether expression of higher levels of ADK enabled enhanced processing of 6-ETI into its active phosphorylated form, which could explain the selectivity of 6-ETI to PEL cells. A panel of representative PELs and non-PELs were examined for ADK expression by immunoblot 24 hours after plating at cell densities used for the viability assays (Figure 5A). Results clearly indicated that PEL cell lines express higher ADK levels than non-PEL, consistent with their heightened sensitivity to 6-ETI. To confirm that this endogenous ADK is necessary to confer sensitivity to 6-ETI, we attempted to knock down ADK in PEL cell lines using lentiviral transduction, but complete suppression was not achieved. Therefore, we used a carcinoma cell line (PA-Tu-8988T), also with high ADK expression, which is more amenable to lentiviral transduction, to test whether genetic suppression of ADK affects sensitivity to 6-ETI. In this experiment, ADK knockdown resulted in a log increase in $\mathrm{LC}_{50}$ (Figure 5B). Thus, ADK activity appears to be a prerequisite for susceptibility to 6-ETI. To further test the model that ADK converts 6-ETI into its monophosphorylated active form (Figure 5C), we synthesized phospho-6-ETI and tested it in PEL and the resistant IBL1 cell line. Figure 5D shows that this modification partially overcomes resistance in IBL1 cells that express low levels of ADK.

We also examined ADK levels for 3 different resistant cell lines at higher plating densities, and found that there was a clear upregulation of ADK with crowding, although this was most robust for the IBL1 cell line, which is an EBV-infected immunoblastic lymphoma (Supplemental Figure 5A). If ADK expression levels were a major determinant of susceptibility to 6-ETI treatment, then sensitivity in non-PEL cell lines may be induced at plating conditions that increase their endogenous ADK levels. To test this hypothesis, we plated resistant IBL1, LY7, and BCKN1 cells at higher cell density $\left(5 \times 10^{5} \mathrm{cells} / \mathrm{ml}\right)$, and performed viability assays using 6-ETI doseresponse curves. The $\mathrm{LC}_{50} \mathrm{~s}$ under these alternate plating conditions decreased strikingly from those determined at the original assay conditions, showing that sensitivity in resistant lymphoma cell lines could be induced by altered cell densities (Supplemental Figure 5B). Taken together, these data show that there is a strong correlation between ADK levels and sensitivity to 6-ETI, explaining the selectivity of PEL cells for 6-ETI-induced toxicity.

We further tested the idea that different experimental conditions, and specifically in vivo growth of some tumor cells as would occur in naturally occurring malignancies, are associated with high ADK expression, which could predict response to 6-ETI treatment. As proof of principle, we examined whether the immunoblastic lymphoma cell line IBL1 expresses ADK in vivo using a mouse xenograft model and assessed tumor responses following treat- 


\section{Table 1. Comparison of 6-ETI with other nucleoside analogs that are FDA approved, using a PEL cell line (BC3), an MM cell line (U266), and an EBV+ DLBCL cell line (IBL1)}

\begin{tabular}{|c|c|c|c|}
\hline $\begin{array}{l}\text { Nucleoside analog } \\
\text { tested }\end{array}$ & $\begin{array}{c}\mathrm{LC}_{50} \mathrm{BC3} \text { (PEL) } \\
(\mathrm{nM})\end{array}$ & $\begin{array}{c}\mathrm{LC}_{50} \mathrm{U}_{(\mathrm{nM})} \\
\text { (MM) }\end{array}$ & $\underset{(\mathrm{nM})}{\mathrm{LC}_{50} \mathrm{IBL1}(\mathrm{DLBCL})}$ \\
\hline 6-ETI & 49.79 & 437.35 & $>>100,000$ \\
\hline Ribavirin & 44,739 & >>>100,000 & 46,487 \\
\hline Vidarabine & $>>100,000$ & $>>100,000$ & $>>100,000$ \\
\hline Didanosine & $>>100,000$ & >>100,000 & $>>100,000$ \\
\hline 6-Mercaptopurine & 5,143 & 10,325 & $>100,000$ \\
\hline Cladribine & 5,237 & 29,192 & 290.2 \\
\hline Fludarabine & $\gg>100,000$ & 90,690 & 28,853 \\
\hline
\end{tabular}

Sensitivity after 48 hours of treatment is shown. Results shown are $\mathrm{LC}_{50} \mathrm{~s}$ from viability assays (CellTiter-Glo) where the mean \pm SEM of 2 independent experiments performed in duplicates is provided.

ment with 6-ETI. We found that this compound reduced the rate of tumor growth in mice, although responses were not as dramatic as those seen in PEL (Supplemental Figure 5C). Ex vivo tumors from these mice demonstrated expression of ADK in a proportion of tumor cells, as evaluated by immunohistochemistry, as well as some apoptosis induced by treatment in vivo, as determined by increased cleaved caspase-3 positivity (Supplemental Figure 5D). Thus, expression of ADK in vivo is associated with partial tumor responses to 6-ETI in an $\mathrm{EBV}^{+}$immunoblastic lymphoma cell line.

$A D K$ expression may be used as a biomarker for sensitivity to 6-ETI. The clear correlation between 6-ETI sensitivity and ADK expression strongly suggested that other malignancies that express ADK may be sensitive to this drug. However, there is little information regarding ADK expression in lymphoid tissues and lymphoid malignancies, and cancer in general, in vivo. We therefore performed immunohistochemistry for ADK in human tonsils, and found that normal plasma cells are positive, based on morphology and costaining with CD138 (Figure 6A). We also confirmed expression of ADK in primary biopsy samples of PEL and extracavitary PEL. Given that PEL most closely resembles plasma cells immunophenotypically $\left(\mathrm{CD} 138^{+}\right)$and by gene expression profiling (30-32), we hypothesized that ADK is highly expressed in other malignancies of plasma cell origin, namely multiple myeloma (MM) and plasmablastic lymphoma. All 4 cases of MM and both cases of plasmablastic lymphoma evaluated showed clear expression of ADK (Figure 6A and Supplemental Figure 6). We therefore evaluated 4 MM cell lines, JJN3, MM1S, U266, and CAG, for their sensitivity to 6-ETI treatment. As was the case for PEL, MM cell lines were sensitive to inhibition by 6-ETI and phospho-6-ETI, with $\mathrm{LC}_{50} \mathrm{~s}$ in the high nanomolar range (Figure 6B and Supplemental Figure 7A). Primary MM samples cultured ex vivo also showed sensitivity to 6-ETI, indicating that this sensitivity is not exclusive to cell lines (Figure 6D). Coculture with bone marrow stromal cells during treatment was assessed for 2 different patient samples, in parallel with separated myeloma cells alone, and there was no significant difference in sensitivity. 6-ETI also suppressed DNA synthesis in MM cells (Figure 6C), and cytotoxicity was associated with induction of apoptosis, autophagy, and activation of a DNA damage response (Supplemental Figure 7B). To assess whether 6-ETI may be toxic to normal plasma cells, we treated total tonsil B lymphocytes with $1 \mu \mathrm{M}$ and $10 \mu \mathrm{M}$ 6-ETI for 24 hours and subsequently gated for plasma cells $\left(\mathrm{CD} 19^{-}, \mathrm{CD}^{-138^{+}}\right)$. We did not find a decrease in the total number or proportion of plasma cells (Figure 6E) upon treatment, so we conclude that while these express high levels of ADK, they are not sensitive, likely because they are not proliferative.

Effectiveness of 6-ETI in a disseminated mouse model of MM. Given the in vitro sensitivity data, we also assessed the antitumor activity of 6-ETI in a disseminated MM xenograft model by injecting NOD/SCID mice i.v. with $\mathrm{Luc}^{+} \mathrm{GFP}^{+} \mathrm{CAG}$ cells stably expressing the HSV-TK-eGFP-luciferase fusion protein. Before injection, we confirmed that CAG-luc cells expressed monotypic cytoplasmic light chains and ADK (Supplemental Figure 7C). Anatomical distribution and pathophysiological manifestation of this model were compatible with the clinical course of MM in humans, i.e., hallmarked by malignant plasma cells present mainly within the bones (sternum, femur, tibia, vertebrae, skull, mandible) and associated with multifocal lytic bone lesions (Supplemental Figure 7C). We monitored tumor burden by bioluminescence imaging. Seven mice were treated with vehicle, and 8 with $200 \mathrm{mg} / \mathrm{kg} / \mathrm{d}$ of 6-ETI (Figure 7A). We found that mice treated with 6-ETI had significant reduction in tumor burden as assessed by the bioluminescent signal (Figure 7, B and C) without any weight loss. Moreover, overall survival was also significantly prolonged in the 6-ETI-treated group compared with the control mice (Figure 7D; $P<0.005$ ).

Comparison with other nucleoside analogs. We tested whether the selectivity profile of 6-ETI to PEL and MM is unique or is a general feature of related nucleoside analogs. We first tested 6-mercaptopurine (6-MP) (Table 1). 6-ETI is structurally similar to 6-MP, but the latter lacks the ribose ring where phosphorylation occurs. Interestingly, 6-MP, while not particularly effective against PEL cells, demonstrated selectivity to them, much like 6-ETI, suggesting that it may be metabolized to an intermediate that may be phosphorylated by ADK, albeit with low efficiency (Table 1). We also tested 4 different purine analogs that are FDA approved: vidarabine, didanosine, cladribine, and fludarabine phosphate. None of these showed the same spectrum of selectivity or effectiveness toward PEL and MM as 6-ETI. To test whether a pyrimidine analog would have an effect toward PEL and MM, we tested ribavirin. This drug has received recent attention for its effect on leukemias acting as a protein synthesis inhibitor, in addition to its antiviral effects (33). However, this drug had an effect on the viability of the 2 lymphoma lines tested only at very high concentrations $\left(\mathrm{LC}_{50}\right.$ $>44 \mu \mathrm{M})$, and even higher for $\mathrm{MM}\left(\mathrm{LC}_{50}>>100 \mu \mathrm{M}\right)$.

\section{Discussion}

This study reports the successful identification of what we believe is the first novel and selective inhibitor of KSHV-associated PEL that clearly exhibits preclinical potential, as evidenced by therapeutically relevant $\mathrm{LC}_{50}$ s and striking effectiveness in vivo in mouse models. In addition, we demonstrated the feasibility of identifying the mechanism of selectivity, in this case activation of the prodrug by endogenous ADK, by using RNA-Seq-based resistome analysis as a methodology that has not, to our knowledge, been previously described for this purpose. We further report expression of ADK in human plasma cells and other lymphoid malignancies of plasma 
cell origin, including MM. We confirmed the relevance of ADK expression and its potential to be used as a biomarker to identify sensitive cancers by documenting in vitro and in vivo responses of MM and PEL to 6-ETI.

The idea that purine analogs function as drugs through their effects on metabolism was proposed in seminal work by Elion and Hitchings in 1965 (34). This class of compounds has been studied over the last 50 years in an effort to develop and improve them as effective anticancer and antiviral agents. In recent years, purine nucleoside analogs (PNAs) have found favor in cancer and antiviral clinical trials. Studies of their mechanisms of action have identified several universal themes. PNAs appear to require phosphorylation for conversion to their active forms within the cell, which can include mono-, di-, or tri-phosphorylation steps. The active compounds have multiple "targets" within the cell. Chiefly, they inhibit metabolism by outcompeting ATP, and inhibiting cellular kinases. Additionally, by dint of their structural similarity, they are usually able to incorporate into DNA and/or RNA, thus leading to chain termination and abrogating synthesis $(35,36)$. A resultant accumulation of double-strand breaks in the cell triggers the apoptotic cascade. By reasons of precedent and as a novel PNA, we propose that 6-ETI is activated by ADK and has deleterious effects on sensitive cells through similar mechanisms, which is supported by our experimental data (Figure 8).

Several pieces of evidence suggested that 6-ETI functions as an antimetabolite in PEL cells. Adenine derivatives with a 6-S substitution have been extensively studied as anticancer and antiviral agents (37-39). The prototypical compound of this class of molecules is 6-mercaptopurine (6-MP) (Table 1$)$, which shares structural similarity with 6-ETI. Since 6-MP is an established antiviral that functions through effects on ribonucleotide pool size (38), it is possible that 6-ETI may also exert effects on nucleotide or nucleoside metabolism. RNA-Seq on 6-ETI-resistant clones was useful to detect changes in gene expression patterns (Supplemental Worksheet 1) that can be used to uncover pathways and signatures. This analysis yielded several folate, purine, and pyrimidine biosynthetic pathways prominently among the top hits by Ingenuity Pathway Analysis (QIAGEN) (Supplemental Table 4 and Supplemental Worksheet 3). However, owing to poor enrichment of nucleotides extracted by conventional methods in PEL cells, possibly attributable to high levels of endogenous nucleotidases, examination of such an effect directly in these cells has been challenging. Additionally, sensitive cell lines showed a dramatic reduction in ATP levels upon 6-ETI treatment, when measured by CellTiter-Glo, consistent with effects observed in other PNAs (Figure 1B). Notably, as part of preliminary experiments to determine the drug target, we cultured resistant cell lines in modified media with stable isotope-labeled arginine and lysine, which caused the $\mathrm{LC}_{50} \mathrm{~s}$ for 6-ETI in these cell lines to plummet to levels observed in sensitive cell lines. In this regard, assessment of RNA expression data showed an enrichment of amino acid metabolic pathways in resistant clones (Supplemental Table 4).

RNA-Seq-based target identification for drugs with unknown mechanisms of action is a novel methodology proposed by Elemento and colleagues (40). This approach was recently used to identify mechanisms of drug resistance for the established anticancer agents ispinesib and YM155 (41), both of which have well-known targets within the cell. To our knowledge, our study is the first to date to effectively demonstrate the use of transcriptome sequencing of drug-resistant clones to determine a definitive mechanism of specificity for a novel lymphoma inhibitor discovered through targeted screening. This is of particular importance in cases of functional screens that identify effective inhibitors with unknown primary targets, such as in this case, wherein the primary highthroughput screen for NF- $\kappa \mathrm{B}$ inhibitors yielded a hit compound that inhibits this pathway as a secondary mechanism. Additionally, the gold standard for target identification, stable isotope labeling of amino acids in cell culture (SILAC), is restricted to molecules that retain activity when structurally modified with a tag, and have a direct molecular target rather than metabolic effects on the cell. Both requirements preclude its utility in the case of 6-ETI. Thus, we demonstrated effective use of a novel methodology to overcome the constraints of existing methods for target identification.

The finding that ADK directly activates 6-ETI by phosphorylation provides an important benchmark in further development of this compound as an anticancer agent, as well as suggesting a probable mechanism of specificity. Specifically, since the normal function of ADK is to phosphorylate adenosine to its monophosphorylated form AMP, thus catalyzing an early step in purine nucleoside biosynthesis, we can postulate a model whereby enhanced ADK expression levels in sensitive cells effectively carry out rapid drug activation, enabling it to reach its "true" target(s) within the cell. In fact, the close structural similarity of 6-ETI to inosine, along with the prevalence of replication/transcription/translation and nucleotide metabolism pathways in the expression analysis, suggests that the drug may act as a subversive substrate for ADK, and thus induce aberrant replication by its inclusion into DNA. We have shown that 6-ETI can significantly affect DNA synthesis using Click-iT EdU assay (Figure 2D and Figure 6C); whether it can directly incorporate into DNA is still to be determined. This possibility of DNA incorporation is supported by the precedent of the nucleoside analog antiviral agents such as acyclovir and ganciclovir, both of which incorporate into viral and cellular DNA to prevent viral replication and cell division (42). The ADK-activated nucleoside analog ribavirin is also known to incorporate into viral nucleic acids (43), and functions primarily through its competition with adenosine and guanosine for dependent processes, such as transcription (44). The only previously published studies on 6-ETI also indicate that the compound effectively inhibits DNA synthesis (45) and replication of RNA viruses (38). Significantly, much like topoisomerase inhibitors, 6-ETI induces $S$ phase arrest in PEL cells, consistent with a direct effect on DNA replication.

The discovery that ADK overexpression enhances sensitivity to 6-ETI immediately expanded the scope of utility for an inhibitor that was initially found to be highly PEL cell-specific. This expectation was reflected in the high expression of ADK in MM tissues and concordant sensitivity of cell lines to this compound (Figure 7). This is consistent with public data in Oncomine (www.oncomine. org), which also shows overexpression of $A D K$ RNA in MM (as the sole hematological malignancy expressing this gene). Encouragingly, recent entrants to the antitumor PNA arsenal such as 8-chloro-adenosine and 8-amino-adenosine have been found to be effective in MM (46-48), directly mediating their effects through a decrease in ATP and DNA and/or RNA synthesis, but these are not 
FDA approved. In fact, among some of the most common purine analogs that are used clinically, none compared to 6-ETI in terms of their selectivity profile. ADK overexpression has also been found in colorectal cancer (49) and astrocytic tumors (50), with $A D K$ gene dysregulation in cancer now emerging as a general theme, and thus, this compound may prove to be effective across a broad range of malignancies. Consequently, ADK expression levels may prove to be an important biomarker for drug response in the future.

While the mechanisms of PNA drug action are fairly universal, many of the newer drugs have been found to possess unique additional properties that may confer greater specificity and pharmacological properties in the clinical context. In fact, even small changes in structure are correlated with different active forms (for example, some do not require phosphorylation), metabolic properties, and mechanisms of action, such as resistance to inhibition by cellular adenosine deaminase (51). A suggestion that 6-ETI may possess a unique mechanism of action arises from our observation that, in contrast to the established mode of PNA-induced cell death, i.e., triggering of the apoptotic cascade, a major mechanism of cell death upon treatment was necrosis. Additionally, while many ADK-dependent nucleoside analogs can also be activated by other cellular kinases, the lack of mutations in other kinase genes found by SNP calling in our resistant clones suggests that ADK may indeed serve as the primary kinase for 6-ETI activation. This notion is supported by increased activity of phospho-6-ETI in a cell line with low levels of ADK.

This study led to the discovery of a new nucleoside analog that is promising for the treatment of plasmacytic aggressive cancers. The survival advantage conferred by 6-ETI in the xenograft mouse models was robust (Figures 3 and 7), indicating that 6-ETI treatment is effective in inhibiting tumor growth. These results, coupled with the therapeutically relevant $\mathrm{LC}_{50}$ s of inhibition observed when PEL and $\mathrm{MM}$ cells were treated with this compound, suggest clear-cut promise for further preclinical development. While there is a reasonable arsenal of drugs for the treatment of MM, resistance to these frequently emerges. In addition, plasmablastic lymphoma is a rare but highly aggressive malignancy for which no effective treatment is available, and the median survival is under 1 year. While ubiquitous expression of ADK is expected based on its important metabolic role, this is not clearly documented, and at least by immunohistochemistry of tonsils, ADK is undetectable in most cells. This may explain the lack of toxicity seen in our mouse models. While a complete toxicological analysis is required, National Cancer Institute assay results from prior testing of this compound revealed it had been shown to exhibit minimal toxicity in other mouse models; it was moderately effective in 1 leukemia model (L1210) but not in other leukemia, adenocarcinoma, or melanoma mouse models. While the basic function of ADK is known, there is not much mechanistic information about its role in cancer, and further biological studies are required. For example, there are nuclear and cytoplasmic isoforms (52), but the specific functional role of these in different cellular compartments is not clear. Mechanistic studies assessing the functional role of ADK in specific cellular compartments are ongoing, and should shed light on the scope of tumors that may respond to 6-ETI.

In this study, we demonstrated the feasibility of identifying a specific inhibitor for herpesvirus-induced tumors and other plasma cell malignancies, including plasmablastic lymphoma, well known to have challenging druggability. The use of unbiased genomics to analyze the resistome of drug-resistant cell lines provides important proof of principle in target identification, especially for compounds failing the narrow structural and functional criteria required by conventional methods such as SILAC. ADK is a relatively unknown player in the field of oncological therapeutics, and its critical role in activation of this new anticancer drug justifies further testing in this context.

We propose that once preclinical testing is completed, 6-ETI is a viable candidate for clinical trials in patients with lymphoid malignancies, using ADK expression as a biomarker. These include PEL, MM, and plasmablastic lymphoma patients, but possibly other hematological malignancies. Additionally, the emerging scenario of ADK expression in other cancer subtypes suggests further promise for this compound in such tumors.

\section{Methods}

Electrophoretic mobility shift assays. BC3 or LCL9001 cells were plated at $5 \times 10^{5}$ cells $/ \mathrm{ml}$, and treated with the hit compounds at $25 \mu \mathrm{M}$ or with NSC39368 at 5, 10, 25, and $50 \mu \mathrm{M}$ for 24 hours. Nuclear extracts were obtained as previously described (53), and EMSAs performed using an $\mathrm{NF}-\kappa \mathrm{B}-$ specific oligonucleotide probe.

Viability assays. Cell viability assays were performed by plating of log-phase cells in RPMI complete media with $20 \%$ FBS at $1 \times 10^{5}$ cells/ $\mathrm{ml}$, with concentrations of 6-ETI or phospho-6-ETI ranging from 0.1 $\mathrm{nM}$ to $100 \mu \mathrm{M}$. ATP content at 24,48 , and 72 hours after treatment was measured by the CellTiter-Glo kit (Promega), or by trypan blue assay as indicated. The $\mathrm{LC}_{50}$ for 6-ETI in each cell line was determined using online half-maximal effective concentration $\left(\mathrm{EC}_{50}\right)$ software from Chang Bioscience (changbioscience.com) or using GraphPad Prism to determine the $\mathrm{LC}_{50}$ for 6-ETI, phospho-6-ETI, and nucleoside analogs in MM cell lines and primary MM patient samples.

Cell cycle and cell death assays. Assessment of cell death in cell lines and primary cells is described in the Supplemental Methods.

DNA synthesis assays. EdU Click-IT assay was used to assess the effects of 6-ETI on DNA synthesis, using BC3 and U266 cells (see Supplemental Methods).

Immunoblotting. For all intracellular signaling studies, cell lines were plated at $2 \times 10^{5}$ cells $/ \mathrm{ml}$ or at indicated densities in RPMI-1640 with $20 \%$ FBS, and treated with the stated concentrations of 6-ETI, from 6 to 48 hours. Immunoblotting was performed using the following antibodies: PARP (BD Pharmingen, 556494), LC3B (Cell Signaling Technology, 2775S), phospho-histone H2AX.X S139 (Cell Signaling Technology, 2577S), GAPDH (Gene Tex, GTX100118), ADK (Santa Cruz Biotechnology, sc-365470), vCyclin (Abcam, ab12208), LANA (Advanced Biotechnologies, 13-210-100), $\beta$-actin (Sigma, A5316), p65 (Cell Signaling Technology, C22B4), p50 (Cell Signaling Technology, 3035), Oct-1 (Cell Signaling Technology, 4428), $\mathrm{pI \kappa B} \alpha$ (Santa Cruz Biotechnology, B-9 sc-8404), IкB $\alpha$ (Santa Cruz Biotechnology, C-21 sc-371), pIKK $\alpha / \beta$ (ser 176) (Santa Cruz Biotechnology, sc-21661), IKK $\alpha$ (Santa Cruz Biotechnology, H-744 sc-7218); vFLIP 6/14 antibody was a gift from Mary Collins, University College of London (London, United Kingdom).

Generation of 6-ETI-resistant cell lines. To generate 6-ETI-resistant clones, BC3 cells were plated in RPMI- 1640 with $50 \mu \mathrm{g} / \mathrm{ml}$ gentamicin and $20 \%$ FBS under 6-ETI selection in multiple 96 -well plates at $1 \times 10^{5}$ cells/ml. Selection was begun at $15 \mathrm{nM}, 30 \mathrm{nM}$, or $60 \mathrm{nM}$ 6-ETI. Cells were split every 3 days, with the dose of 6-ETI doubled at least 1 time 
per week. Selection continued until a few clonal populations remained, after which the clones were expanded into fresh plates under selection with $10 \mu \mathrm{M}$ 6-ETI. Clones retrieved were designated NSCE-1, NSCE-2, NSCE-3, NSCE-4, NSCE-5, NSCE-6, NSCE-7, NSCE-8, in chronological order of selection from the original plates. NSCE- 4 and NSCE- 5 ceased growth and were not included in the final analysis.

Additionally, in 2 mice in the low-dose cohort of the xenograft trial (see below), tumors grew out beyond the 100-day trial cessation time point. The ascites from these mice were harvested ex vivo and, following Ficoll extraction, placed in culture in RPMI-1640 with $10 \%$ FBS. After 6-ETI viability assays determined these xenograft cell lines, designated 2-3low and 3-5low, to have decreased sensitivity to 6-ETI, they were placed under bulk selection in RPMI-1640 with 20\% FBS and $10 \mu \mathrm{M}$ 6-ETI to generate resistance. Retesting determined the outgrowing cells to be completely resistant to 6-ETI $\left(\mathrm{LC}_{50} \mathrm{~s}>100 \mu \mathrm{M}\right)$, and these were subsequently included in RNA-Seq analysis.

RNA sequencing. RNA was extracted using the RNeasy Mini Kit (Qiagen). Illumina libraries were constructed from RNA with the Illumina Truseq Total RNA Library Preparation Kit (v2) and sequenced on Illumina HiSeq in single-end 51-bp mode. Raw data contained 50 million to 64 million reads, or a total of 2,600-3,300 gigabases, per sample. The methods used to analyze the RNA sequencing data are provided as Supplemental Methods. RNA-Seq data are available in the NCBI's BioProject database under accession number PRJNA362820.

Computational approach to 3D modeling of ADK WT and mutant proteins (G239E and G243K). The x-ray crystallographic structure of human adenosine kinase (ADK) (1BX4) was used as the initial structure with 2 adenosine molecules, one bound to its substrate-binding site and the other to its ATP-binding site (38). To prepare the WT enzyme model, Protein Preparation Wizard (Schrödinger) (54) was used for the addition of missing residues and atoms, removal of the adenosine molecule in the ATP-binding site, removal of bad contacts, optimization of bond lengths, and removal of all ions and water molecules. The structure was further optimized by the addition of hydrogen atoms using Schrödinger's Maestro interface. The prepared model was then used to generate 2 mutant structures, G239E and G243K, using Maestro. The adenosine molecule in the substrate-binding site was modified using Maestro to resemble 6-ETI. Six models of ADK were prepared to illustrate the positioning of the mutated residues with respect to either the bound adenosine or 6-ETI molecules.

In vitro kinase assay. The ADK kinase assay kit was purchased from Novocib, France. ADK and ATP provided in the kit were premixed into provided kinase assay buffer. The reaction at $37^{\circ} \mathrm{C}$ was started by addition of 6-ETI in DMSO at $t=0$, followed by quenching of $10 \mu \mathrm{l}$ of the reaction by $30 \mu \mathrm{l}$ acetonitrile in an Eppendorf tube at 5, 10, 15, $30,60,120,150,180$, and 360 minutes. The quenched reaction was briefly vortexed and centrifuged at approximately 7,000 $\mathrm{g}$, and then the supernatant was transferred into a fresh tube and stored at $4^{\circ} \mathrm{C}$ for HPLC/MS analysis. A control reaction was set up containing ADK, ATP, and inosine, in order to generate a standard peak for IMP, while ADK, DTT, and ATP controls were generated by dissolving of individual kit components in a 3:1 acetonitrile/ $\mathrm{dH}_{2} \mathrm{O}$ solution.

HPLC/MS analysis for 6-ETI phosphorylation by ADK. LC/MS separations were performed using a Waters ACQUITY UPLC system equipped with ACQUITY Photodiode Array (PDA), a Waters Micromass SQD 2000 spectrometer, and a Waters ACQUITY BEH C18 column $(1.7 \mu \mathrm{m}, 2.1 \times 100 \mathrm{~mm})$. The solvent system consisted of $0.1 \%$ formic acid in water (solvent $\mathrm{A}$ ) and $0.1 \%$ formic acid in acetonitrile (solvent B). Flow was set to $0.3 \mathrm{ml} / \mathrm{min}$, and a gradient of $5 \%$ to $95 \%$ solvent B was applied over a period of 3 minutes. Total run time was 4 minutes. Eluents were detected using a PDA at a wavelength of 254 $\mathrm{nm}$. Mass data were obtained in both positive and negative electrospray mode, at a cone voltage of $30 \mathrm{~V}$.

In vivo xenograft models. The $\mathrm{BC} 3$ in vivo xenograft model was set up as previously described (6). Essentially, approximately 4to 6-week-old male NOD/SCID mice (Jackson Laboratory, stock 001303) were injected i.p. with $1 \times 10^{7}$ BC3NFRen-luc\#3 cells and followed by in vivo imaging every 3 days. After imaging on day 5 to confirm establishment of the tumor, the mice were randomized to vehicle, low-dose, and high-dose cohorts, with average tumor burden distributed evenly across the groups. Each dose cohort contained 10 mice. Mice were treated for 9 days beginning at randomization with vehicle (PBS-Tween-80), low-dose 6-ETI (150 mg/kg/d), or high-dose 6-ETI $(300 \mathrm{mg} / \mathrm{kg} / \mathrm{d})$. We monitored mice for tumor growth by live imaging and weighing, with the sacrifice point determined to be a net gain or loss of $10 \%$ body weight over a week. Upon reappearance of refractory tumors, mice were retreated for a further 9-day interval, and followed for a total of 100 days. We assessed compound effects on overall survival, progression-free survival, and tumor burden by Kaplan-Meier curves generated using GraphPad Prism software, and determined $P$ values by 2-tailed analysis with log-rank tests.

For the disseminated MM xenograft model, CAG cells were provided by the Malcolm Moore laboratory at Memorial Sloan Kettering Cancer Center. $1 \times 10^{7}$ CAG cells stably expressing the HSV-TKeGFP-luciferase fusion protein were injected i.v. into NOD/SCID mice (NOD/LTSZPrko/J, Jackson Laboratory, stock 001303). Eight days after tumor induction, mice were randomized into control and treated cohorts and treated i.p. with either PBS-0.05\% Tween-80 (vehicle) or $200 \mathrm{mg} / \mathrm{kg}$ of 6-ETI daily for 9 days. Tumor mass or bioluminescence (photons $/ \mathrm{s} / \mathrm{cm}^{2} /$ steradian) was evaluated by imaging of the mice using an IVIS Imaging system. Statistical analysis was done using a 2-tailed, unpaired Student's $t$ test with GraphPad prism.

Statistics. In all cases in which 2 groups were compared, unpaired 2-tailed Student's $t$ tests or log-rank tests were used to determine significance. A $P$ value less than 0.05 was considered statistically significant. All bar and line graphs show mean \pm SEM of 3 experiments.

Study approval. These studies were approved by the Weill Cornell Medical College IACUC.

\section{Author contributions}

$\mathrm{UN}$ and JS designed and executed most experiments and cowrote the paper. DHH, GA, and JT performed additional experiments. JR performed single-nucleotide variant and gene expression analysis on RNA-Seq data. HZ performed pathway analysis on gene expression data. MAS modeled ADK binding; PJB synthesized 6-ETI and performed HPLC/MS. IG assisted with growth curves on cells. DJ and RN processed and provided the primary MM patient specimens. DH analyzed preliminary gene expression data. YL performed immunohistochemistry on primary tumors and IBL1 xenografts. SS and RHS supervised, designed, and executed high-throughput screening. JDW designed and supervised 6-ETI and p6-ETI synthesis and HPLC/MS. OE supervised singlenucleotide variant and gene expression analysis. KMK supervised some experiments and edited the paper. EC supervised and 
designed all experiments, analyzed gene expression data, and cowrote and edited the paper.

\section{Acknowledgments}

The authors thank Weimin Zhu and Andrea Gardner for technical assistance and Joseph Tomaszewski for critical advice. Immunofluorescence was performed at the Rockefeller University Bioimaging Resource Center. This project has been funded with federal funds from the NIH, under grant R01CA154228 to EC and through the Inter-Institute Program for the Development of AIDS-Related Therapeutics (IIP). This project has also been funded in whole or in part with federal funds from the National Cancer Institute (NCI), NIH, under contract HHSN261200800001E, as well as NCI grant CA082036 to KMK. UN was funded by training grant T32AI007621. The content of this publication does not necessarily reflect the views or policies of the Department of Health and Human Services, nor does mention of trade names, commercial products, or organizations imply endorsement by the US Government.

Address correspondence to: Ethel Cesarman, Department of Pathology and Laboratory Medicine, Weill Cornell Medical Col- lege, 1300 York Avenue, New York, New York 10065, USA. Phone: 212.746.8838; E-mail: ecesarm@med.cornell.edu.

UN's present address is: Department of Medical Oncology, DanaFarber Cancer Institute, Boston, Massachusetts, USA.

JR's present address is: Memorial Sloan Kettering Cancer Center, New York, New York, USA.

DHH's present address is: Department of Biology, Farmingdale State College, Farmingdale, New York, USA.

MAS's present address is: Department of Life Sciences, Whitelands College, University of Roehampton, London, United Kingdom.

SS and RHS's present address is: Chemopreventive Agent Development Research Group, Division of Cancer Prevention, National Cancer Institute, Bethesda, Maryland, USA.

JT's present address is: Chapman University School of Pharmacy, Biomedical and Pharmaceutical Sciences, Orange, California, USA.
1. Chang Y, et al. Identification of herpesvirus-like DNA sequences in AIDS-associated Kaposi's sarcoma. Science. 1994;266(5192):1865-1869.

2. Soulier J, et al. Kaposi's sarcoma-associated herpesvirus-like DNA sequences in multicentric Castleman's disease. Blood. 1995;86(4):1276-1280.

3. Cesarman E, Chang Y, Moore PS, Said JW, Knowles DM. Kaposi's sarcoma-associated herpesvirus-like DNA sequences in AIDS-related body-cavity-based lymphomas. N Engl J Med. 1995;332(18):1186-1191.

4. Sarek G, et al. Reactivation of the $\mathrm{p} 53$ pathway as a treatment modality for KSHV-induced lymphomas. JClin Invest. 2007;117(4):1019-1028.

5. Nayar U, et al. Targeting the Hsp90-associated viral oncoproteome in gammaherpesvirus-associated malignancies. Blood. 2013;122(16):2837-2847.

6. Keller SA, et al. NF-kappaB is essential for the progression of KSHV- and EBV-infected lymphomas in vivo. Blood. 2006;107(8):3295-3302.

7. Guasparri I, Keller SA, Cesarman E. KSHV vFLIP is essential for the survival of infected lymphoma cells. J Exp Med. 2004;199(7):993-1003.

8. Liu L, Eby MT, Rathore N, Sinha SK, Kumar A, Chaudhary PM. The human herpes virus 8-encoded viral FLICE inhibitory protein physically associates with and persistently activates the IкB kinase complex. J Biol Chem. 2002;277(16):13745-13751.

9. Field N, et al. KSHV vFLIP binds to IKK- $\gamma \backslash$ to activate IKK. J Cell Sci. 2003;116(pt 18):3721-3728.

10. Guzman JR, Koo JS, Goldsmith JR, Mühlbauer M, Narula A, Jobin C. Oxymatrine prevents NF- $\kappa$ B nuclear translocation and ameliorates acute intestinal inflammation. Sci Rep. 2013;3:1629.

11. Pérez $\mathrm{M}$, et al. The fungal metabolite galiellalactone interferes with the nuclear import of NF- $\mathrm{KB}$ and inhibits HIV-1 replication. Chem Biol Interact. 2014;214:69-76.

12. McCall K. Genetic control of necrosis - another type of programmed cell death. Curr Opin Cell
Biol. 2010;22(6):882-888.

13. Ewald B, Sampath D, Plunkett W. Nucleoside analogs: molecular mechanisms signaling cell death. Oncogene. 2008;27(50):6522-6537.

14. Chandriani S, Ganem D. Array-based transcript profiling and limiting-dilution reverse transcription-PCR analysis identify additional latent genes in Kaposi's sarcoma-associated herpesvirus. J Virol. 2010;84(11):5565-5573.

15. Vieira J, O'Hearn PM. Use of the red fluorescent protein as a marker of Kaposi's sarcoma-associated herpesvirus lytic gene expression. Virology. 2004;325(2):225-240.

16. Boison D, et al. Neonatal hepatic steatosis by disruption of the adenosine kinase gene. Proc Natl Acad Sci U S A. 2002;99(10):6985-6990.

17. Newby AC. The role of adenosine kinase in regulating adenosine concentration. Biochem J. 1985;226(1):343-344.

18. Berne RM. Adenosine - a cardioprotective and therapeutic agent. Cardiovasc Res. 1993;27(1):2.

19. Boison D. Adenosine dysfunction in epilepsy. Glia. 2012;60(8):1234-1243.

20. Willis RC, Carson DA, Seegmiller JE. Adenosine kinase initiates the major route of ribavirin activation in a cultured human cell line. Proc Natl Acad Sci U S A. 1978;75(7):3042-3044.

21. Wu JZ, Larson G, Walker H, Shim JH, Hong Z. Phosphorylation of ribavirin and viramidine by adenosine kinase and cytosolic 5'-nucleotidase II: Implications for ribavirin metabolism in erythrocytes. Antimicrob Agents Chemother. 2005;49(6):2164-2171.

22. Mori $\mathrm{K}$, et al. Adenosine kinase is a key determinant for the anti-HCV activity of ribavirin. Hepatology. 2013;58(4):1236-1244.

23. Ptak RG, et al. Phosphorylation of triciribine is necessary for activity against HIV type 1 . AIDS Res Hum Retroviruses. 1998;14(15):1315-1322.

24. Koyama H, Tsuji M. Genetic and biochemical studies on the activation and cytotoxic mecha- nism of bredinin, a potent inhibitor of purine biosynthesis in mammalian cells. Biochem Phar macol. 1983;32(23):3547-3553.

25. Saunders PP, Spindler CD, Tan MT, Alvarez E, Robins RK. Tiazofurin is phosphorylated by three enzymes from Chinese hamster ovary cells. Cancer Res. 1990;50(17):5269-5274.

26. Gallois-Montbrun S, et al. Structural analysis of the activation of ribavirin analogs by NDP kinase: comparison with other ribavirin targets. Mol Pharmacol. 2003;63(3):538-546.

27. McGaraughty S, et al. Effects of A-134974, a novel adenosine kinase inhibitor, on carrageenaninduced inflammatory hyperalgesia and locomotor activity in rats: evaluation of the sites of action. JPharmacol Exp Ther. 2001;296(2):501-509.

28. Jarvis MF, et al. ABT-702 (4-amino-5-(3-bromophenyl)-7-(6-morpholinopyridin-3-yl)pyrido[2, 3-d]pyrimidine), a novel orally effective adenosine kinase inhibitor with analgesic and anti-inflammatory properties: I. In vitro characterization and acute antinociceptive effects in the mouse. JPharmacol Exp Ther. 2000;295(3):1156-1164.

29. Wotring LL, Crabtree GW, Edwards NL, Parks RE, Townsend LB. Mechanism of activation of triciribine phosphate (TCN-P) as a prodrug form of TCN. Cancer Treat Rep. 1986;70 (4):491-497.

30. Gaidano G, et al. Association of Kaposi's sarcomaassociated herpesvirus-positive primary effusion lymphoma with expression of the CD138/syndecan-1 antigen. Blood.1997;90(12):4894-4900.

31. Klein U, et al. Gene expression profile analysis of AIDS-related primary effusion lymphoma (PEL) suggests a plasmablastic derivation and identifies PEL-specific transcripts. Blood. 2003;101(10):4115-4121.

32. Jenner RG, et al. Kaposi's sarcoma-associated herpesvirus-infected primary effusion lymphoma has a plasma cell gene expression profile. Proc Natl Acad Sci U S A. 2003;100(18):10399-10404.

33. Borden KL. Targeting the oncogene eIF4E in can- 
cer: From the bench to clinical trials. Clin Invest Med. 2011;34(6):E315.

34. Elion GB, Hitchings GH. Metabolic basis for the actions of analogs of purines and pyrimidines. Adv Chemother. 1965;2:91-177.

35. Elion GB. The biochemistry and mechanism of action of acyclovir. JAntimicrob Chemother. 1983;12(Suppl B):9-17.

36. Plunkett W, Huang P, Gandhi V. Metabolism and action of fludarabine phosphate. Semin Oncol. 1990;17(5 Suppl 8):3-17.

37. Montgomery JA, Johnston TP, Gallagher A, Stringfellow CR, Schabel FM. A comparative study of the anticancer activity of some S-substituted derivatives of 6-mercaptopurine and their ribonucleosides. JMed Pharm Chem. 1961;3:265-288.

38. Yamamoto K, Hasobe M, Saneyoshi M. Inhibitory effect of sulphur-containing purine nucleoside analogues on replication of RNA viruses: selective antiviral activity against influenza viruses. Acta Virol. 1988;32(5):386-392.

39. Saneyoshi M, Hasobe M, Yamamoto K. Antiviral activities and the mechanism of 9-beta-Dribofuranosyl-6-alkylthiopurines on several RNA viruses from animals. Nucleic Acids Symp Ser. 1989;(21):49-50.

40. Wacker SA, Houghtaling BR, Elemento O, Kapoor TM. Using transcriptome sequencing to identify mechanisms of drug action and resis- tance. Nat Chem Biol. 2012;8(3):235-237.

41. Kasap C, Elemento O, Kapoor TM. DrugTargetSeqR: a genomics- and CRISPR-Cas9-based method to analyze drug targets. Nat Chem Biol. 2014;10(8):626-628.

42. De Clercq E, Neyts J. Antiviral agents acting as DNA or RNA chain terminators. Handb Exp Pharmacol. 2009;(189):53-84.

43. Cameron CE, Castro C. The mechanism of action of ribavirin: lethal mutagenesis of RNA virus genomes mediated by the viral RNAdependent RNA polymerase. Curr Opin Infect Dis. 2001;14(6):757-764.

44. Jordan I, Briese T, Averett DR, Lipkin WI. Inhibition of Borna disease virus replication by ribavirin. JVirol. 1999;73(9):7903-7906.

45. Kuwano M, Matsui K, Nakashima T, Endo H, Komiyama S. Potentiation of cytostatic effect of sulfur- or chlorine-containing purines and related ribonucleosides by amphotericinB on cultured mouse leukemia cells. Gan. 1975;66(6):655-661.

46. Gandhi V, Ayres M, Halgren RG, Krett NL, Newman RA, Rosen ST. 8-chloro-cAMP and 8-chloro-adenosine act by the same mechanism in multiple myeloma cells. Cancer Res. 2001;61(14):5474-5479.

47. Krett NL, et al. 8-amino-adenosine is a potential therapeutic agent for multiple myeloma. $\mathrm{Mol}$
Cancer Ther. 2004;3(11):1411-1420.

48. Frey JA, Gandhi V. 8-Amino-adenosine inhibits multiple mechanisms of transcription. Mol Cancer Ther. 2010;9(1):236-245.

49. Giglioni S, et al. Adenosine kinase gene expression in human colorectal cancer. Nucleosides Nucleotides Nucleic Acids. 2008;27(6):750-754

50. de Groot M, et al. Overexpression of ADK in human astrocytic tumors and peritumoral tissue is related to tumor-associated epilepsy. Epilepsia. 2012;53(1):58-66.

51. Jordheim LP, Durantel D, Zoulim F, Dumontet C. Advances in the development of nucleoside and nucleotide analogues for cancer and viral diseases. Nat Rev Drug Discov. 2013;12(6):447-464.

52. Cui XA, Singh B, Park J, Gupta RS. Subcellular localization of adenosine kinase in mammalian cells: The long isoform of AdK is localized in the nucleus. Biochem Biophys Res Commun. 2009;388(1):46-50.

53. Keller SA, Schattner EJ, Cesarman E. Inhibition of NF- $\kappa \mathrm{B}$ induces apoptosis of KSHV-infected primary effusion lymphoma cells. Blood. 2000;96(7):2537-2542.

54. Sastry GM, Adzhigirey M, Day T, Annabhimoju $\mathrm{R}$, Sherman W. Protein and ligand preparation: parameters, protocols, and influence on virtual screening enrichments. JComput Aided Mol Des. 2013;27(3):221-234. 